\title{
A Study of Generalized Lienard Equation using Modified Homotopy Perturbation Method
}

\author{
${ }^{1}$ Mohammed S.Mechee and ${ }^{1}$ Ghassan A. Al-Juaifri and ${ }^{2}$ Yasen Rajihy \\ ${ }^{1}$ Department of Mathematics, Faculty of Computer Science and Mathematics, \\ Kufa University, Najaf, Iraq \\ ${ }^{2}$ Department of Software, Faculty of Information Technology, University of Babylon, Hilla, Iraq
}

\begin{abstract}
In this study, we have studied a class of nonlinear Lienard equation which is used as mathematical models in many physically significant fields and applied science. The Homotopy Perturbation Method (HPM) has been modified for solving generalized linear and nonlinear Lienard equation and the Duffing equation which is a non-linear second-order differential equation and usually used to model certain damped and driven oscillators. Also, we have tested the modified HPM on the solving of different implementations which show the efficiency and accuracy of the proposed method. The approximated solutions of HPM agree well with analytical solutions for the tested problems.
\end{abstract}

Key words: Lienard equation, homotopy, HPM, ordinary differential equations, ODE, proposed method

\section{INTRODUCTION}

The most important mathematical models for physical phenomena is the differential equation. Motion of objects, fluid and heat flow, bending and cracking of materials, vibrations, chemical reactions and nuclear reactions are all modeled by systems of differential equations. Moreover, numerous mathematical models in science and engineering are expressed in terms of unknown quantities and their derivatives. Many applications of Differential Equations (DEs), particularly ODEs of different orders, can be found in the mathematical modeling of real life problems (Mechee et al., 2014).

The Homotopy Perturbation Method (HPM) which are a well-known is efficient technique to find the approximate solutions for ordinary and partial differential equations which describe different fields of science, physical phenomena, engineering, mechanics and so on. HPM was proposed by He (1999) for solving linear and nonlinear differential equations and integral equations. Many researchers used HPM to approximate the solutions of differential equations and integral equations (Yildirim, 2010; Jalaal et al., 2010; Ma et al. 2008).

Many researchers published some papers on solving some classes differential equations using HPM. For example, Chun and Sakthivel (2010) used HPM for solving a linear and non-linear second-order two-point boundary value problems while Gulkac (2010) was solved the Black-Scholes equation for a simple European option in this method to obtain a new efficient recurrent relation to solve Black-Scholes equation. Moreover, numerous researches used HPM for solving nonlinear differential equations, Vahidi et al. (2011) was solved non-linear DEs which yields the Maclaurin series of the exact solution, (Chang and Liou, 2006) developed a third-order explicit approximation to find the roots of the dispersion relation for water waves that propagate over dissipative media (Zhou and $\mathrm{Wu}, 2012$ ) solved the non-linear PB equation describing spherical and planar colloidal particles immersed in an arbitrary valence and mixed electrolyte solution, Ozis and Akci (2011) solved certain non-linear, non-smooth oscillators, Yazdi (2013) solved nonlinear vibration analysis of functionally graded plate while $\mathrm{He}$ (2004) applied HPM for solving nonlinear oscillators with discontinuities, nonlinear Duffing equation and some nonlinear ODEs. For class of linear partial differential equations, Al-Saif and Abood (2011) solved the Korteweg-de Vries (KdV) equation and convergence study of HPM, Babolian et al. (2009) used HPM to solve time-dependent differential equations Aswhad and Jaddoa (2016) solved advection problem, vibrating beam equation linear and nonlinear PDEs and the system of nonlinear PDEs and Babolian et al. (2009) used the homotopy perturbation method to solve timedependent differential. Also, many researchers used HPM for solving the class of non-linear PDEs, Yazdi (2013) was approximated solution for free nonlinear

Corresponding Author: Mohammed S. Mechee, Department of Mathematics, Faculty of Computer Science and Mathematics, Kufa University, Najaf, Iraq 
vibration of thin rectangular laminated FGM plates, Liao (2004) has solved nonlinear PDEs, Yildirim (2009) was used to implement the nonlinear Korteweg-de Vries equation, Md Nasrudin et al. combined HPM-Pade approximant to acquire the approximate analytical solution of the KdV equation, Taghipour (2010) solved parabolic equations and periodic equation linear and nonlinear PDEs, Janalizadeh et al. obtained the solution of a second-order non-linear wave equation, Fereidoon et al. (2011) utilized to derive approximate explicit analytical solution for the nonlinear foam drainage equation, Momani and Odibat (2007) modified the algorithm which provides approximate solutions in the form of convergent series with easily computable components, Babolian et al. (2009) solved time-dependent differential equations while $\mathrm{He}(2000)$ solved non-linear problems using the homotopy technique. However, for the system of Des, Bataineh et al. (2009) solved systems of second-order BVPs, Javidi (2009) solved SEIR Model, Wang and Song (2007) solution of a model for HIV infection of CD4+T cells, Rafei et al. (2007) solution of the system of nonlinear ordinary differential equations governing on the problem, Noor et al. (2013) solved the system of linear equations. Noor and Mohyud-Din (2008) solution of linear and non-linear sixth-order boundary value problems and system of differential equations, Javidi (2009) solved system of linear Fredholm Integral Equations (LFIEs). Yusufogclu (2009) has solved a linear fredholm type integro-differential equations with separable kernel. Javidi (2009) solved non-linear Fredholm integral equations, Saberi-Nadjafi and Tamamgar (2008) used modified HPM for solving the system linear and nonlinear of Volterra integral equations Kumar et al. (2011) solved generalized Abel integral equation. Lastly for the differential equations of fractional type, Odibat and Momani (2008) solved nonlinear differential equations of fractional order, Jafari et al. (2010) solved nonlinear problems of fractional Riccati differential equation and Yildirim and Agirseven (2009) solved the spacetime fractional advection-dispersion equation. Lastly, Mechee et al. (2017 b) have studied second-order PDEs using HPM while Mechee et al. (2017a) modified HPM for solving generalized linear complex differential equations.

Recently, we have studied a wide class of nonlinear Lienard equation which are used as mathematical models in many physically significant fields and applied science. The approximated solutions of this class of differential equations have studied using modefided Homotopy Perturbation Method (HPM). Also, the approximated solution of Duffing equation which is a non-linear second-order differential equation and usually used to model certain damped and driven oscillators has been obtained. The proposed method is applied for solving different examples for this class of ordinary differential equations. A comparison was made between the analytical and approximated solutions for different tested problems which showed the proposed method is more efficient in the iterations complexity and high accurate in the absolute errors. It has been highlighted that the use of modified HPM is more suitable to approximate the solutions of general Lienard and Duffing differential equations with considering the general coefficients functions.

\section{Preliminary}

Lienard Equation: In this research, we consider the general Lienard equation which has the following form (Mat-infar et al., 2008; Abbasbandy et al., 2011; Harko et al., 2014):

$$
x^{\prime}(t)+f(x)(t) x(t)+g(x(t))=h(t), x(t) \in R
$$

which is not only regarded as a generalization of the damped pendulum equation or a damped spring-mass system (where $f(x(t)) x(t)$ is the damping force, $g(x(t))$ is the restoring force and $h(t)$ is the external force) but also, used as nonlinear models in many physically significant fields when taking different choices for $\mathrm{f}(\mathrm{xt})$ ), $g(x(t))$ and $h(t)$. For example, the choices $f(x(t))=\left(x^{1} 1\right)$, $g(x(t))=x(t)$ and $h(t)=0$ lead Eq. 1 to the Van der Pol equation served as a nonlinear model of electronic oscillation. Therefore, studying Eq. 1 is of physical significance. In the general case it is commonly believed that it is very difficult to find its exact solution by usual ways (Harko et al., 2014)

$$
x(t)+1 x(t)+m x^{3}(t)+n x^{5}(t)=0
$$

Some researchers studied the following special case of Eq. 2 where 1, $m$ and $n$ are real coefficients. Finding explicit exact and numerical solutions of nonlinear equations efficiently is of major importance and has widespread applications in numerical methods and applied mathematics. In this study, we will implement the (HPM) to find the approximate solutions to the Lienard equation. An other example of Lienard equation is given by the Duffing's equation, The Duffing equation, named after Georg Duffing is a non-linear second-order differential 
equation used to model certain damped and driven oscillators (Matinfar et al., 2008; Chun and Sakthivel 2010). The Duffing equation is given by:

$$
\mathrm{x}(\mathrm{t})+(\mathrm{ax}(\mathrm{t})+\mathrm{b}) \mathrm{x} \cdot(\mathrm{t})+\mathrm{cx}(\mathrm{t})-\mathrm{x}^{3}(\mathrm{t})+\mathrm{d}=0
$$

where, a-d are constants and $x(t)$ is proportional to a uniquely specified similarity length scale Lienard-type equations also, naturally appear in the mathematical description of some important astrophysical phenomena equation is obtained by subjecting stationary solutions of spherically symmetric accretion processes to time-dependent radial perturbations and including nonlinearities of any arbitrary order. From a physical point of view, the Lienard equation represents a generalization of the equation of damped oscillations,

$$
\ddot{x}(t)+\gamma \dot{x}(t)+w^{2} x(t)=0
$$

where, $r$ and $\mathrm{w}$ are constant parameters. For $\mathrm{r}=0$, we obtain the equation of the linear harmonic oscillator which represents one of the fundamental equations of both classical and quantum physics. Generally, a linear oscillation can be described by the following equation (Harko et al., 2014):

$$
x(t)+f(x(t)) x(t)+g(x(t)) x(t)=0
$$

In particular it has been found a polynomial $\mathrm{f}(\mathrm{x})$ of ${ }^{\circ} 6$ such that the associated Lienard equation has at least four limit cycles. Thus, there are no general results about the limit cycles when $f(x)$ is a polynomial of degree greater than 5 neither in general whenf $(x)$ is an arbitrary real function and a variational method showing that limit cycles correspond to relative extrema of certain functionals is explained in here, we are interested in the application of another technique, the Homotopy analysis method (HPM), to this problem. The generalized method have been applied in the weakly nonlinear regime of the Lienard equation. The essential difference of these methods with the HAM is that this last method introduces a new perturbation parameter that allows to go further in the validity respect to the physical parameter for the solutions of the system (Fereidorn et al., 2011).

Homotopy Perturbation Method (HPM): In this study, we present a brief description of the HPM, to illustrate the basic ideas of the homotopy perturbation method, we consider the following differential equation (Neamaty and Darzi, 2010; Chun and Sakthivel, 2010, Batiha, 2015; Abbasbandy, 2006):

$$
\mathrm{A}(\mathrm{u}) \mathrm{f}(\tau)=0, \quad \tau \in \Omega
$$

with boundary conditions:

$$
\frac{\partial \mathrm{u}}{\mathrm{B}(\mathrm{u}, \partial \tau)=0} \quad \tau \in \partial \Omega
$$

Where:

$\mathrm{A}=$ General differential operator

$\mathrm{B}=\mathrm{A}$ boundary operator

$\mathrm{f}(\cdot)=$ A known analytic function

$\infty=$ The boundary of the domain.

The operator A can be generally divied into two parts of $\mathrm{L}$ and $\mathrm{N}$ where $\mathrm{L}$ is linear part while $\mathrm{N}$ is the nonlinear part in the DE, therefore, Eq. 6 can be rewritten as follows (He, 1999):

$$
\mathrm{L}(\mathrm{u})+\mathrm{N}(\mathrm{u})-\mathrm{f}(\tau)=0
$$

By using homotopy technique, one can construct a homotopy, $\mathrm{V}(, \mathrm{p}) \mathrm{:}_{\mathrm{e}} \times[0,1], \mathrm{R}$ which satisfies:

$$
\begin{aligned}
& H(v, p)=(1-p)\left[L(v)-L\left(u_{0}\right)\right]+p[L(v)+N(v)-f(\tau)]=0 \\
& H(v, p)=L(v)-L\left(u_{0}\right)+p L\left(u_{0}+p[N(v)-f(\tau)]\right)=0
\end{aligned}
$$
or:

where, $p_{\bullet}[0,1], \ldots$ and $p$ is called homotopy parameter and $\mathrm{u}$. is an initial approximation for the solution of Eq. 6 which satisfies the boundary conditions obviously, using Eq. 9 or 10, we have the following Eq. 11:

and:

$$
\mathrm{H}(\mathrm{v}, 0)=\mathrm{L}(\mathrm{v})-\mathrm{L}\left(\mathrm{u}_{0}\right)=0
$$

$$
\mathrm{H}(\mathrm{v}, 1)=\mathrm{L}(\mathrm{v})+\mathrm{N}(\mathrm{v})-\mathrm{f}(\tau)=0
$$

Assume that the solution of Eq. 9 or 10 can be expressed as a series in $\mathrm{p}$ as follows:

$$
\mathrm{V}=\mathrm{v}_{0}+\mathrm{p}_{\mathrm{v} 1}+\mathrm{p}_{\mathrm{v} 2}^{2}+\mathrm{p}_{\mathrm{v} 3}^{3}+\ldots=\sum_{\mathrm{i}=1}^{\infty} \mathrm{p}^{\mathrm{i} \mathrm{v}^{\mathrm{i}}}
$$

setp .1; results in the approximate solution of (Eq. 6). Consequently:

$$
\mathrm{u}(\tau)=\lim _{\mathrm{p} \rightarrow 1} \mathrm{~V}=\mathrm{v}_{0}+\mathrm{v}_{2}+\mathrm{v}_{3}+\ldots \mathrm{v}_{\mathrm{i}}=\sum_{\mathrm{i}=0}^{\infty} \mathrm{v}_{\mathrm{i}}
$$

It is worth to note that the major advantage of He's homotopy perturbation method is that the perturbation equation can be freely constructed in many ways and approximation can also, be freely selected.

Analysis of modified HPM for solving Lienard differential equation: In this study, we will present a 
proposed of the homotopy perturbation method to solving Lienard equations, we consider the following equation:

$$
\mathrm{x}(\mathrm{t})+\mathrm{f}(\mathrm{x}) \dot{\mathrm{x}}(\mathrm{t})+\mathrm{g}(\mathrm{x})=\mathrm{h}(\mathrm{t}), \quad \mathrm{t}_{0} \leq \mathrm{t} \leq \mathrm{t}_{1}
$$

with th einitial conditions:

$$
\begin{aligned}
& x\left(t_{0}\right)=\alpha \\
& x\left(t_{0}\right)=\beta
\end{aligned}
$$

where, and Bare arbitrary constants (Matinfar et al., 2008; Harko et al., 2014). The implementation of proposed modefied HPM for solving Lineard differential Eq. 15 according the following algorithm:

\section{MATERIALS AND METHODS}

Firstly, we start with the initial approximation $\mathrm{x} 0(\mathrm{t})={ }_{\mathrm{a}}+\mathrm{t}_{\mathrm{t}} \mathrm{t}$, Secondly, we can construct a homotopy for second-order ODE (Eq. 15) as follow:

$$
\begin{aligned}
& H(x, p)=(1-p)\left(x-x_{0}\right)+ \\
& p(x \cdot(t)+f(x) x(t)+g(x)-h(t))=0
\end{aligned}
$$

Thirdly, using Taylor expansion for the functions $f$ and $g$ about $t=0$, by substituting the Taylor expansions for the coefficients function $f(x)=\sum_{i=0}^{\infty} a_{i} x^{i}, g(x)=\sum_{i=0}^{\infty} b_{i} x^{i}$ and $h(t)=\sum_{i=0}^{\infty} c_{i} t^{i}$ coefficents. However:

$$
\begin{aligned}
& H(x, p)=(i-p)\left(\sum_{i=0}^{\infty} x_{i} p^{i}-x_{0}\right)+ \\
& p\left(\sum_{i=0}^{\infty} x_{i} p^{i}+\sum_{i=0}^{\infty} a_{i} x^{i}+\sum_{i=0}^{\infty} b_{i} x^{i}\right)
\end{aligned}
$$

consequently, we substitute $x(t)=\sum_{k=0}^{\infty} p^{k} x_{k}$ in the Eq. 19 to get the following form:

$$
\begin{aligned}
& \mathrm{H}(\mathrm{x}, \mathrm{p})=(1-\mathrm{p})\left(\sum_{\mathrm{i}=0}^{\infty} \mathrm{x}_{i} \mathrm{p}^{\mathrm{i}-\mathrm{x}_{0}}\right)+ \\
& \mathrm{p}\left(\sum_{\mathrm{i}=0}^{\infty} \mathrm{x}_{i} \mathrm{p}^{\mathrm{i}}+\sum_{\mathrm{i}=0}^{\infty} \mathrm{a}_{i}\left(\sum_{\mathrm{k}=0}^{\infty} \mathrm{x}_{\mathrm{k}} \mathrm{p}^{\mathrm{k}}\right)^{\mathrm{I}} \sum_{\mathrm{i}=0}^{\infty} \mathrm{b}_{\mathrm{i}} \mathrm{x}^{\mathrm{i}}+\right. \\
& \left.\sum_{i=0}^{\infty} \mathrm{b}_{i}\left(\sum_{\mathrm{i}=0}^{\infty} \mathrm{x}_{\mathrm{k}} \mathrm{p}^{\mathrm{k}}\right)-\mathrm{h}(\mathrm{t})\right)=0
\end{aligned}
$$

Fourthly, suppose that the solution of Eq. 15 is in the form:

$$
\mathrm{x}(\mathrm{t})=\mathrm{x}_{0}+\mathrm{p}_{\mathrm{x} 1}+\mathrm{p}_{\mathrm{x} 2}^{2}+\mathrm{p}_{\mathrm{x} 3}^{3}+, \ldots
$$

Fifthly, collecting terms of the same power of $\mathrm{p}$ gives, as show in the following equations:

$$
\begin{aligned}
& P^{\prime}: \ddot{x}_{1}(t)+\ddot{x}_{0}(t)+\sum_{=0}^{\infty} a_{1} x_{0}^{i}(t) \ddot{x}(t)+\sum_{=0}^{\infty} b_{1} x_{0}^{1}(t)-h(t)=0 \\
& P^{2}: \ddot{x}_{2}(t)+\sum_{=0}^{\infty} a_{1} \dot{x}_{0}(t) \ddot{x}_{1}(t)+\sum_{=0}^{\infty} i a_{1} x_{0}^{i-1}(t) x_{1} x_{0}(t)+ \\
& \sum_{=0}^{\infty} i b_{1} x_{0}^{j-1}(t) x_{1} x_{0}(t)=0 \\
& P^{3}: \ddot{x}_{3}(t)+\sum_{=0}^{\infty} a_{1} x_{0}^{i}(t)+x_{1}(t) x^{1(t)} \sum_{=0}^{\infty} i a_{1} x_{0}^{i-1}(t)+ \\
& x_{0}(t) x_{2}(t) \sum_{=0}^{\infty} i a_{1} x_{0}^{j-1}(t)+x_{0}(t) x_{1}^{2}(t) \sum_{=2}^{\infty} a x_{0}^{i-2}(t)+ \\
& \mathrm{x}_{2}(\mathrm{t}) \sum_{-1}^{\infty} \mathrm{ib} \mathrm{x}_{0}^{\mathrm{i}-1}(\mathrm{t})+\mathrm{x}_{1}^{2} \sum_{=2}^{\infty} \mathrm{b}_{1} \mathrm{a}_{\mathrm{i}}^{\mathrm{i}-2}(\mathrm{t}) \\
& P^{A}: \ddot{x}_{4}(t)+\dot{x}_{0}(t) x_{3}(t) \sum_{=1}^{\infty} i a_{1} x_{0}^{i-1}(t) \dot{x}_{0}(t) x_{1}(t) x_{2}(t) \sum_{=3}^{\infty}\left(i^{2}-1\right) a_{1} x_{0}^{i}(t)+ \\
& \dot{\mathrm{x}}_{0}(\mathrm{t}) \mathrm{x}^{3}(\mathrm{t}) \sum_{=3}^{\infty} \mathrm{Ba}_{1} \mathrm{x}_{0}^{\mathrm{i}-3}(\mathrm{t})+
\end{aligned}
$$

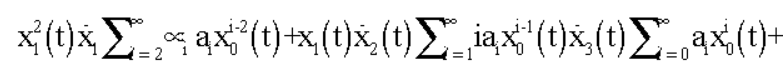

$$
\begin{aligned}
& x_{3}(t) \sum_{=0}^{\infty} i b_{1} x_{0}^{i-1}(t)+x_{1}(t) x_{3}(t) \sum_{=2}^{\infty}\left(i^{2}-1\right) b_{1} x_{0}^{i-2}(t)+ \\
& \mathrm{x}_{1}^{3}(\mathrm{t}) \sum_{=3}^{\infty} \mathrm{B}_{1} \mathrm{~b}_{1}^{\mathrm{i}-3}(\mathrm{t})=0
\end{aligned}
$$

And:

$$
\begin{aligned}
& \mathrm{P}^{5}: \ddot{\mathrm{x}}_{5}(\mathrm{t})+\ddot{\mathrm{x}}_{4}(\mathrm{t})+\ddot{\mathrm{x}}_{1}(\mathrm{t}) \sum_{\mathrm{i}=0}^{\infty} \mathrm{a}_{\mathrm{i}} \mathrm{x}_{0}^{\mathrm{i}}(\mathrm{t})+\dot{\mathrm{x}}_{3}(\mathrm{t}) \mathrm{x}_{1}(\mathrm{t}) \sum_{i=1}^{\infty} \mathrm{ia}_{\mathrm{i}} \mathrm{x}_{0}^{\mathrm{i}-1}(\mathrm{t})+ \\
& \dot{\mathrm{x}}_{2}(\mathrm{t}) \mathrm{x}_{3}(\mathrm{t}) \sum_{\mathrm{i}=1}^{\infty} \mathrm{ia} \mathrm{x}_{0}^{\mathrm{i}-1}(\mathrm{t})+\dot{\mathrm{x}}_{2}(\mathrm{t})+\mathrm{x}_{2}^{2}(\mathrm{t}) \sum_{\mathrm{i}=2}^{\infty} \infty_{\mathrm{i}} \mathrm{a}_{1} \mathrm{x}_{0}^{\mathrm{i}-2}(\mathrm{t})+ \\
& \ddot{x}_{1}(t) x_{3} \sum_{i=1}^{\infty} a_{1} x_{0}^{i-1}(t)+\ddot{x}_{1}(t) x_{1}(t) x_{2}(t) \sum_{i=2}^{\infty}\left(i^{2}-1\right) a_{i} x_{0}^{i-2}(t)+ \\
& \ddot{\mathrm{x}}_{1}(\mathrm{t}) \mathrm{x}_{1}^{3} \sum_{\mathrm{i}=3}^{\infty} \mathrm{B}_{1} \mathrm{a}_{\mathrm{i}} \mathrm{x}_{0}^{\mathrm{i}-3}(\mathrm{t})+0(\mathrm{t}) \mathrm{x}_{4}(\mathrm{t}) \sum_{\mathrm{i}=1}^{\infty} \mathrm{ia}_{\mathrm{i}} \mathrm{x}_{0}^{\mathrm{i}-1}(\mathrm{t})+ \\
& \dot{x}_{0}(t) x_{1}(t) x_{3}(t) \sum_{i=2}^{\infty}\left(i^{2}-1\right) a_{i} x_{0}^{i-2}(t)+ \\
& \dot{\mathrm{x}}_{0}(\mathrm{t}) \mathrm{x}_{2}^{2} \sum_{\mathrm{i}=2}^{\infty} \mathrm{B}_{1} \mathrm{a}_{\mathrm{i}} \mathrm{x}_{0}^{\mathrm{i}-2}(\mathrm{t})+\mathrm{O}(\mathrm{t}) \mathrm{x}_{1}^{2}(\mathrm{t}) \mathrm{x}_{2}(\mathrm{t}) \sum_{\mathrm{i}=3}^{\infty} \infty_{\mathrm{i}} \mathrm{a}_{\mathrm{i}} \mathrm{i}_{0}^{\mathrm{i} \cdot 3}(\mathrm{t})+ \\
& \dot{x}_{0}(t) x_{1}^{4}(t) \sum_{i=4}^{\infty} w_{i} a_{i} x_{0}^{i-4}(t)+x_{4}(t) \sum_{i=3}^{\infty} i b_{i} x_{0}^{i-1}(t)+ \\
& \mathrm{x}_{1}(\mathrm{t}) \mathrm{x}_{3}(\mathrm{t}) \sum_{\mathrm{i}=2}^{\infty}\left(\mathrm{i}^{2}-1\right) \mathrm{b}_{\mathrm{i}} \mathrm{x}_{0}^{\mathrm{i}-2}(\mathrm{t})+ \\
& \mathrm{x}_{2}^{2}(\mathrm{t}) \sum_{\mathrm{i}=2}^{\infty} \mathrm{B}_{1} \mathrm{~b}_{\mathrm{i}} \mathrm{x}_{0}^{\mathrm{i}-2}(\mathrm{t}) \mathrm{x}_{1}^{2}(\mathrm{t}) \sum_{\mathrm{i}=3}^{\infty} \partial_{\mathrm{i}} \mathrm{b}_{\mathrm{i}} \mathrm{x}_{0}^{\mathrm{i}-3}(\mathrm{t})+ \\
& \mathrm{x}_{1}^{4}(\mathrm{t}) \sum_{\mathrm{i}=4}^{\infty} \mathrm{w}_{\mathrm{i}} \mathrm{b}_{\mathrm{i}} \mathrm{x}_{0}^{\mathrm{i} 4}(\mathrm{t})=0 \\
& \text {." }=1,3,6,10,15, \ldots \text {, } \\
& \text { p. }=1,4,10,20, \ldots \\
& \text {. }=3,12,30,60, \ldots \text {, } \\
& \mathrm{w} .=1,5,15, \ldots
\end{aligned}
$$

Finally, using Eq. 22 with some simplifications, we get the following terms of the solution: 
$\mathrm{x}_{0}(\mathrm{t})=\alpha+\beta \mathrm{t}$

$\mathrm{x}_{1}(\mathrm{t})=-\iint_{\mathrm{t}} \ddot{\mathrm{x}}_{0}(\mathrm{~s})+\sum_{i=1}^{\infty} \mathrm{a}_{1} \mathrm{x}_{0}(\mathrm{~s})^{\mathrm{I}} \dot{\mathrm{x}}_{0}(\mathrm{~s})+\sum_{=0}^{\infty} \mathrm{b}_{1} \mathrm{x}_{0}(\mathrm{~s})^{\mathrm{I}} \mathrm{h}(\mathrm{s}) \mathrm{dsdt}$

$x_{2}(t)=-\iint_{t}\left(\sum_{i=0}^{\infty} a_{1} x_{0}^{1}(t) \dot{x}_{1}(t)+\sum_{i=1}^{\infty} i_{1} x_{0}^{i-1}(t) x_{1} \dot{x}_{0}(t)+\right.$

$\left.\sum_{i=1}^{\infty} i b_{1} x_{0}^{i-1}(t) x_{1}(t)\right) d s d t$

$X_{s}(t)=-\iint_{t} \dot{x} 2(t) \sum_{=0}^{\infty} a_{1} x_{0}^{i}(t)+\dot{x}_{1}(t) \sum_{=1}^{\infty} i_{1} x_{0}^{i-1}(t)+$

$\dot{x}_{0}(t) x_{2}(t) \sum_{=1}^{\infty} a_{i} x_{0}^{i-1}(t)+x 0(t) x_{1}^{2}(t) \sum_{=2}^{\infty} a_{1} x_{0}^{i-2}(t)+$

$\mathrm{x}_{2}(\mathrm{t}) \sum_{=1}^{\infty} \mathrm{bb}_{1} \mathrm{~b}_{0}^{\mathrm{j}-1}(\mathrm{t})+\mathrm{x}_{1}^{2} \sum_{i=0}^{\infty} \mathrm{b}_{1} \mathrm{a}_{1} \mathrm{x}_{0}^{\mathrm{j}-2}(\mathrm{t}) \mathrm{dsdt}$

$\mathrm{X}_{4}(\mathrm{t})=-\iint_{\mathrm{t}} \mathrm{x} 0(\mathrm{t}) \mathrm{x}_{3}(t) \sum_{=1}^{\infty} \mathrm{ia}_{1} \mathrm{x}_{0}^{\mathrm{i}-1}(\mathrm{t}) \mathrm{x} \mathrm{x}(\mathrm{t}) \mathrm{x}_{2}(\mathrm{t}) \sum_{=2}^{\infty}\left(\mathrm{i}^{2}-1\right) \mathrm{a}_{1} \mathrm{x}_{0}^{\mathrm{i}-2}(\mathrm{t})+$

$\dot{x}_{0}(t) x_{1}^{3}(t) \sum_{=3}^{\infty} B_{1} a_{1}^{i-3}(t)+\dot{x} l(t) x_{2}(t) \sum_{=1}^{\infty} i a_{1} x_{0}^{i-1}(t)+$

$\mathrm{x}_{1}^{2}(\mathrm{t}) \dot{\mathrm{x}} 1 \sum_{=2}^{\infty} \alpha_{1}^{\infty} \mathrm{a}_{1} \mathrm{x}_{0}^{\mathrm{i}-2}(\mathrm{t})+\mathrm{x}_{1}(\mathrm{t}) \dot{\mathrm{x}} \mathrm{x}(\mathrm{t}) \sum_{=1}^{\infty} \mathrm{ia}_{\mathrm{i}} \mathrm{x}_{0}^{\mathrm{i}-1}(\mathrm{t})+$

$\dot{x}_{0}(t) \sum_{=0}^{\infty} a x_{0}^{i}(t)+x_{3}(t) \sum_{=1}^{\infty} i b_{1} x_{0}^{j-1}(t)+x_{1}(t) x_{3}(t) \sum_{=2}^{\infty}\left(i^{2}-1\right) b_{1} x_{0}^{i-2}(t)+$

$\mathrm{x}_{1}^{3}(\mathrm{t}) \sum_{=3}^{\infty} \mathrm{B}_{1} \mathrm{~b}_{1} \mathrm{x}_{0}^{\mathrm{i}-3}(\mathrm{t}) \mathrm{dsdt}$

$\mathrm{x}_{\mathrm{s}}(\mathrm{t})=-\iint_{\mathrm{t}} \dot{\mathrm{x}} 4(\mathrm{t}) \sum_{=0}^{\infty} \mathrm{a} \mathrm{x}_{0}^{\mathrm{i}}(\mathrm{t})+\dot{\mathrm{x}} 3 \mathrm{x}_{1}(\mathrm{t}) \sum_{i=1}^{\infty} \mathrm{ia} \mathrm{x}_{0}^{\mathrm{i}-1}(\mathrm{t})+$

$\dot{x}_{2}(t) x_{3} \sum_{=1}^{\infty} i_{1} x_{0}^{i-1}(t)+\dot{x} 2(t) x_{2}^{2}(t) \sum_{=2}^{\infty} \alpha_{1}^{\infty} a x_{0}^{i-2}(t)+$

$\dot{x}_{1}(t) \sum_{=1}^{\infty} i_{1} x_{1} x_{0}^{i-1}(t)+\dot{x} 1(t) x_{1}(t) x_{2}(t) \sum_{i=2}^{\infty}\left(i^{2}-1\right) a_{1} x_{0}^{i-2}(t)+$

$\dot{x}_{1}(t) x_{1}^{3} \sum_{=3}^{\infty} \mathrm{Ba}_{1} x_{0}^{\mathrm{i}-3}(t)+0(t) x_{4}(t) \sum_{=1}^{\infty} \mathrm{ia}_{1} \mathrm{x}_{0}^{\mathrm{i}-1}(\mathrm{t})+$

$\dot{x}_{0}(t) x_{1}(t) x_{3}(t) \sum_{=2}^{\infty}\left(i^{2}-1\right) a_{i} x_{0}^{i-2}(t) \dot{x} 0(t) x_{2}^{2} \sum_{=2}^{\infty} B_{1} a_{i} x_{0}^{i-2}(t)+$

$\dot{x}_{0}(t) x_{1}^{2}(t) x_{2}(t) \sum_{i=3}^{\infty} \partial_{1} b_{1} x_{0}^{i \cdot 3}(t)+\dot{x} 0(t) x_{1}^{4}(t) \sum_{i=4}^{\infty} w_{i} b_{i} x_{0}^{i-4}(t)+$

$\left.\mathrm{x}_{4}(\mathrm{t})\right) \sum_{=1}^{\infty} b_{1} \mathrm{x}_{0}^{\mathrm{i}-1}(\mathrm{t})+\mathrm{x}_{1}(\mathrm{t}) \mathrm{x}_{3}(\mathrm{t}) \sum_{i=2}^{\infty}\left(\mathrm{i}^{2}-1\right) \mathrm{b}_{1} \mathrm{x}_{0}^{\mathrm{i}-2}(\mathrm{t})+$

$\mathrm{x}_{2}^{2}(\mathrm{t}) \sum_{=2}^{\infty} \mathrm{B}_{1} \mathrm{~b}_{1} \mathrm{x}_{0}^{\mathrm{i}-2}(\mathrm{t})+\mathrm{x}_{1}^{2}(\mathrm{t}) \mathrm{x}_{2}(\mathrm{t}) \sum_{=3}^{\infty} \partial_{1} \mathrm{~b}_{\mathrm{i}} \mathrm{x}_{0}^{\mathrm{i}-3}(\mathrm{t})+$

$\left.\left.x_{1}^{4}(t) \sum_{=4}^{\infty} w_{i} b_{1} x_{0}^{i 4}(t)\right)\right) d s d t$

consequently, the general solution of Eq. 15 is given as follow:

$$
x(t)=x_{0}(t)+x_{1}(t)+x_{2}(t)+x_{3}(t) x_{4}(t)+x_{5}(t)+, \ldots
$$

\section{RESULTS AND DISCUSSION}

Implementation: In order to assess the accuracy of the solving generalized Lienard equation by Homotopy Perturbation Method (HPM) we will introduce some different examples in general and to compare the approximated solution with the exact solutions for these problems, we will consider the following six problems.

Problem 1 (Sturm-Liouville equation): The SturmLiouville equation defined as follows: Neamaty and Darzi (2010).

$$
x(t)-\lambda x(t)+t=0
$$

Subject to the initial conditions:

$$
\mathrm{x}(0)=\mathrm{a}, \mathrm{x}(0)=\mathrm{b}
$$

And the exact solution given by:

$$
x(t)=C e^{\frac{\gamma}{\lambda t}}+C 2 e^{-\frac{\lambda}{\lambda t}}+\frac{t}{\lambda}
$$

Comparing Eq. 25, we have:

$$
\begin{aligned}
& f(x)=0, g(x)=-\lambda x(t), x(t)= \\
& b_{1} x, b_{1}=-\lambda, i=1, \text { and } h(t)=-t
\end{aligned}
$$

The initial approximation has the following form:

$$
\mathrm{x}_{0}(\mathrm{t})=\mathrm{a}+\mathrm{bt}
$$

Substituting Eq. 23 intoEq. 25, we have:

$$
\begin{gathered}
\mathrm{x}_{\mathrm{I}}(\mathrm{t})=-\iint_{\mathrm{t}}((-\mathrm{h})(\mathrm{a}+\mathrm{bs})+\mathrm{s}) \mathrm{dsdt} \\
=\alpha \lambda \frac{\mathrm{t}^{2}}{2 !}+(\mathrm{b} \lambda-1) \frac{\mathrm{t}^{2}}{3 !} \\
\mathrm{X}_{\mathrm{k}}(\mathrm{t})=\iint_{2} \lambda_{\mathrm{Xk}-1}(\mathrm{~s}) \mathrm{ds} \mathrm{dt} ; \mathrm{k}=2,3,4, \ldots
\end{gathered}
$$

So, simplification of Eq. 29 lends to the following solutions:

$$
\begin{aligned}
& \mathrm{X}_{2}(\mathrm{t})=\iint_{2} \lambda \mathrm{x}_{1}(\mathrm{~s}) \mathrm{dsdt}=\mathrm{a} \lambda^{2}-\frac{\mathrm{t}^{4}}{4 !}+\left(\mathrm{b} \lambda^{2}-\lambda\right) \frac{\mathrm{t}^{5}}{5 !} \mathrm{x}_{3} \mathrm{t}= \\
& \iint_{2} \lambda_{\mathrm{x} 2}(\mathrm{~s}) \mathrm{dsdt}=\mathrm{a} \lambda-\frac{\mathrm{t}^{6}}{6 !}\left(\mathrm{b} \lambda^{3}-\lambda^{2} \frac{\mathrm{t}^{7}}{7 !}\right) \mathrm{x}_{\mathrm{k}}(\mathrm{t})= \\
& \mathrm{a} \lambda^{\mathrm{k}} \frac{\mathrm{t}^{2} \mathrm{k}}{2 \mathrm{k} !}+\left(\mathrm{b} \lambda^{\mathrm{k}}-\lambda^{\mathrm{k}} \frac{\mathrm{t}^{2}+1}{(2 \mathrm{k}+1) !}\right.
\end{aligned}
$$

Then, the general solution is given as follow (Fig. 1):

$$
\begin{aligned}
& \mathrm{xl}(\mathrm{t})=\mathrm{x}_{0}(\mathrm{t})+\mathrm{xl}(\mathrm{t})+\mathrm{x}_{2}(\mathrm{t})+\mathrm{x}_{3}(\mathrm{t})++, \ldots,+\mathrm{xk}(\mathrm{t})+ \\
& =\mathrm{a}+\mathrm{bt}+\mathrm{a} \lambda \frac{\mathrm{t}^{2}}{2 !}+(\mathrm{b} \lambda-1) \frac{\mathrm{t}^{3}}{3 !}+\mathrm{a} \lambda^{2} \frac{\mathrm{t}^{4}}{4 !}+\left(\mathrm{b} \lambda^{2}-\lambda\right) \frac{\mathrm{t}^{5}}{5 !}+\mathrm{a} \lambda^{3} \frac{\mathrm{t}^{6}}{6 !} \\
& +\left(\mathrm{b} \lambda^{3}-\lambda^{2}\right) \frac{\mathrm{t}^{7}}{7 !}+\ldots,+\mathrm{a} \lambda^{\mathrm{k}} \frac{\mathrm{t}^{2} \mathrm{k}}{2 \mathrm{k} !}+\mathrm{a} \lambda^{\mathrm{k}}-\lambda \mathrm{k}-1 \frac{\mathrm{t}^{2} \mathrm{k}+1}{(2 \mathrm{k}+1) !}+\ldots
\end{aligned}
$$

Problem 2 (linear Lienard equation): The linear Lienard equation is defined as follow: 


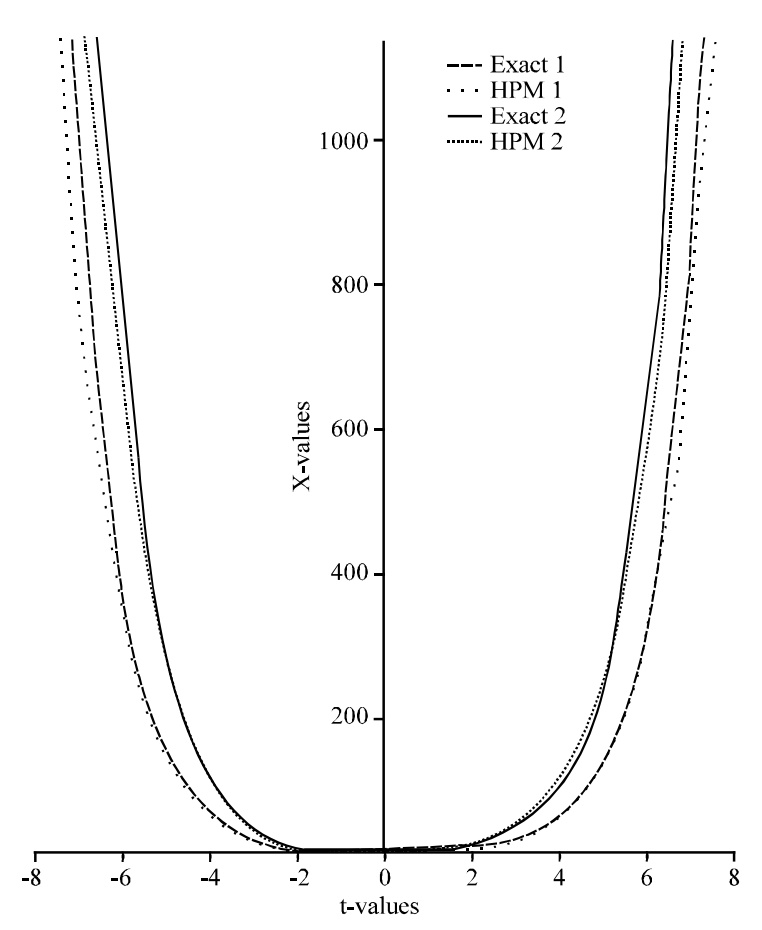

Fig. 1: Comparison of proposed HPM and exact solutions of problem 1 at $\mathrm{c}=\mathrm{c}=1 ; 2=1 ; \mathrm{a}=2 ; \mathrm{b}=1$ and at $\mathrm{c}$. $=\mathrm{c}_{1}=2 ;{ }_{2}=1 ; \mathrm{a}=4 ; \mathrm{b}=1$

$$
\ddot{\mathrm{x}}(\mathrm{t})+\dot{\mathrm{x}}(\mathrm{t})+\mathrm{x}(\mathrm{t})=0
$$

Subject to initial conditions:

$$
x(0)=1, \dot{x}(0)=\frac{\sqrt{3}}{2}-\frac{1}{2}
$$

With the exact solution:

$$
x(t)=C e^{\frac{-1}{2} t} \sin \left(\frac{1}{2} t \sqrt{3}\right)+C 2 e^{\frac{-1}{2} t} \cos \left(\frac{1}{2} t \sqrt{3}\right.
$$

Comparing Eq. 30 we have $f(x)=1=a, i=0, g(x)=x(t)$ $=\mathrm{bx}, \mathrm{b}=1, \mathrm{i}=1$ and $\mathrm{h}(\mathrm{t})=0$. The initial approximation has the form:

$$
\mathrm{x}_{0}(\mathrm{t})=1+\left(\frac{\sqrt{3}}{2}-\frac{1}{2}\right) \mathrm{t}
$$

Substituting Eq. 23 into Eq. 30, we have:

$$
\begin{aligned}
& X_{1}(t)=-\iint_{t}\left(\ddot{x}_{0}(s)+\dot{x}_{0}(s)+x_{0}(s)\right) d s \\
& =\left(\frac{-\sqrt{3}}{2}-\frac{1}{2}\right) \frac{t^{2}}{2 !}\left(\frac{\sqrt{3}}{2}-\frac{1}{2}\right) \frac{t^{3}}{3 !}
\end{aligned}
$$

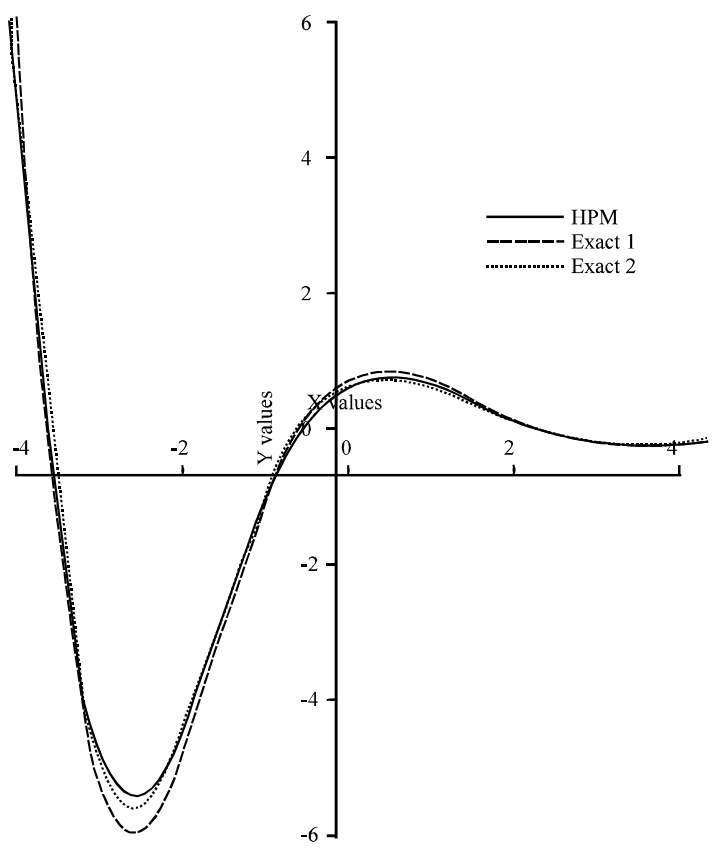

Fig. 2: Comparison of proposed HPM and exact solutions of problem 2 at $\mathrm{c}=\mathrm{c}_{\mathrm{s}}=1$ and $\mathrm{c}=\mathrm{c}_{\mathrm{s}}=1.1$

$$
\mathrm{X}_{\mathrm{k}}=-\iint_{\mathrm{t}}\left(\mathrm{x}_{\mathrm{k}-1}(\mathrm{~s})+\mathrm{X}_{\mathrm{k}-1}(\mathrm{~s})\right) \mathrm{ds} \mathrm{dt} ; \mathrm{k}=2,3,4, \ldots
$$

Consequently, simplification of Eq. 33 leads to the following solutions:

$$
\begin{aligned}
& \mathrm{x}_{2}(\mathrm{t})=-\iint_{\mathrm{t}}\left(\mathrm{x}_{1}(\mathrm{~s})+\mathrm{x}_{1}(\mathrm{~s})\right) \mathrm{ds} \mathrm{dt}= \\
& -\left(\frac{\sqrt{3}}{2}-\frac{1}{2}\right) \frac{\mathrm{t}^{3}}{3 !}+\sqrt{3} \frac{\mathrm{t}^{4}}{4 !}+\left(\frac{\sqrt{3}}{2}-\frac{1}{2}\right) \frac{\mathrm{t}^{5}}{5 !} \\
& \mathrm{X}_{3}(\mathrm{t})=-\iint_{\mathrm{t}}\left(\mathrm{x}_{2}(\mathrm{~s})+\mathrm{x}_{2}(\mathrm{~s})\right) \mathrm{ds} \mathrm{dt}= \\
& \left(\frac{-\sqrt{3}}{2}-\frac{1}{2}\right) \frac{\mathrm{t}^{3}}{3 !}-\sqrt{3 \frac{\mathrm{t}^{5}}{5 !}}-\left(\frac{3 \sqrt{3}}{2}-\frac{1}{2}\right) \frac{\mathrm{t}^{6}}{6 !}-\left(\frac{\sqrt{3}}{2}-\frac{1}{2}\right) \frac{\mathrm{t}^{7}}{7 !} \\
& \mathrm{x}_{4}(\mathrm{t})=-\iint_{\mathrm{t}}(\mathrm{t}) \mathrm{x}_{3}(3)+(\mathrm{s}) \mathrm{x}_{3}(\mathrm{~s}) \mathrm{ds} \mathrm{dt}= \\
& \left.\int\left(-\frac{-\sqrt{3}}{2}-\frac{1}{2}\right) \frac{\mathrm{t}^{4}}{4 !}+\frac{-\sqrt{3}}{2}-\frac{1}{2}\right) \frac{\mathrm{t}^{5}}{5 !}-\left(\frac{-5 \sqrt{3}}{2}-\frac{1}{2}\right) \frac{\mathrm{t}^{6}}{6 !}- \\
& (2 \sqrt{3}-1) \frac{\mathrm{t}^{7}}{7 !}+\left(\frac{\sqrt{3}}{2}-\frac{\mathrm{t}^{9}}{!}\right)
\end{aligned}
$$

Then, the general solution is given as follow (Fig. 2):

$$
\begin{aligned}
& x(t)=x_{0}(t)+x_{1}(t)+x_{2}(t)+x_{3}(t)+, \ldots,+x_{k}(t)+= \\
& 1+\left(\frac{\sqrt{3}}{2}-\frac{1}{2}\right) t-\left(\frac{\sqrt{3}}{2}-\frac{1}{2}\right) \frac{t^{2}}{2 !}+\left(\frac{\sqrt{3}}{2}-\frac{1}{2}\right) \frac{t^{4}}{4 !}+ \\
& \left(\frac{-\sqrt{3}}{2}-\frac{1}{2}\right) \frac{t^{5}}{5 !}+\frac{t^{6}}{6 !}+\left(\frac{\sqrt{3}}{2}-\frac{1}{2}\right) \frac{t^{7}}{7 !}+
\end{aligned}
$$




$$
\begin{aligned}
& \sum_{\mathrm{n}=0}^{\infty} \frac{\left(\frac{-\mathrm{t}}{2}\right)^{\mathrm{n}}}{\mathrm{n} !}\left(\sum_{\mathrm{n}=0}^{\infty}(-1)\right) \frac{\mathrm{n}\left(\frac{\sqrt{3 \mathrm{t}}}{2}\right)^{2 \mathrm{n}}}{2 \mathrm{n} !}+ \\
& \sum_{\mathrm{n}=0}^{\infty}(-1) \frac{\mathrm{n}\left(\frac{\sqrt{3 \mathrm{t}}}{2}\right)^{2 \mathrm{n}+1}}{(2 \mathrm{n}+1) !}+\mathrm{e}^{\frac{-1}{2}}\left(\cos \frac{\sqrt{3 \mathrm{t}}}{2}+\sin \frac{\sqrt{3 \mathrm{t}}}{2}\right)
\end{aligned}
$$

Problem 3 (non linear Lienard equation): Consider the following Van der pol oscillator equation as example for non linear Lienard equation which defined as follow (Abbasbandy et al., 2011):

$$
\ddot{x}(t)+\mu\left(x^{2}(t)-1\right) x \cdot(t)=0
$$

subject to initial conditionis. From Eq. 34, we get $x(0)=1$, $x(0)=1 f(x)=\mu\left(x^{\prime}-1\right)=a+a, x, a=-\mu, a,=\mu, g(x)=x=d x, d$. $=1, \mathrm{~h}(\mathrm{t})=0$ suppose the initial approximation:

$$
x_{0}(t)=1+t
$$

$\mathrm{x}_{1}(\mathrm{t})=\left(\ddot{\mathrm{x}}(\mathrm{s})+\mu \dot{\mathrm{x}}_{0}(\mathrm{~s})\left(\mathrm{x}^{2}(\mathrm{~s})-1\right)+\mathrm{x}_{0}(\mathrm{~s})\right) \mathrm{dsdt}$

$\left.\mathrm{X}_{1}(\mathrm{t})=-\iint_{\mathrm{t}} \ddot{\mathrm{x}}_{0}(\mathrm{~s})+\mu \dot{\mathrm{x}}_{0}(\mathrm{~s})\left(\mathrm{x}_{0}^{2}(\mathrm{~s})-1\right)+\mathrm{x}_{0}(\mathrm{~s})\right) \mathrm{dsdt}=$

$\frac{-1}{12} \mu \mathrm{t}^{4}-\left(\frac{\mu}{3}+\frac{1}{6}\right) \tau^{3}-\frac{1}{2} \tau^{2} \mathrm{X}_{2}(\mathrm{t})=-\iint_{\mathrm{t}}-\mu \ddot{\mathrm{x}}_{1}(\mathrm{~s})+$

$\left.\mu \mathrm{x}_{0}^{2}(\mathrm{~s}) \dot{\mathrm{x}}_{1}(\mathrm{~s})+2 \mu \mathrm{x}_{0}(\mathrm{~s}) \mathrm{x}_{1}(\mathrm{~s}) \dot{\mathrm{x}}_{0}(\mathrm{~s})+\mathrm{x}_{1}(\mathrm{~s})\right) \mathrm{dsdt}=$

$\frac{1}{84} \mu^{2} t^{7}+\frac{1}{360}\left(30 \mu^{2}+11 \mu\right) \tau^{6}+\frac{1}{240}\left(32 \mu^{2}+44 \mu+2\right) \tau^{5}+$

$\frac{1}{144}(36 \mu+6) \tau^{4} \mathrm{X}_{3}(\mathrm{t})=-\iint_{\mathrm{t}}\left(\left(-\mu \dot{\mathrm{x}}_{2}(\mathrm{~s})+\mu \mathrm{x}_{0}^{2}(\mathrm{~s}) \dot{\mathrm{x}}_{2}(\mathrm{~s})+\right.\right.$

$2 \mu \mathrm{x}_{0}(\mathrm{~s}) \mathrm{x}_{1}(\mathrm{~s}) \mathrm{x}_{1}(\mathrm{~s})+2 \mu \mathrm{x}_{0}(\mathrm{~s}) \mathrm{x}_{2}(\mathrm{~s}) \mathrm{x}_{0}(\mathrm{~s})+\mu \mathrm{x}_{1}^{2}(\mathrm{~s}) \mathrm{x}_{0}(\mathrm{~s})+$

$(\mathrm{s}))) \mathrm{dsdt}=-\frac{19}{10080} \mu^{3} \mathrm{t}^{10}-\frac{1}{120960}\left(2280 \mu^{3}+804 \mu^{2}\right) \tau^{9}-$

$\frac{1}{94080}\left(5488 \mu^{3}+5628 \mu^{2}+476 \mu\right) \tau^{8}-$

$\frac{1}{70560}\left(3808 \mu^{3}+11760 \mu^{2}+2856 \mu+14\right) \tau^{7}-$

$\frac{1}{50400}\left(7000 \mu^{2}+4620 \mu+70\right) \tau^{6}-\frac{1}{20} \mu \mathrm{t}^{5} \mathrm{X}_{4}(\mathrm{t})=$

$-\iint_{\mathrm{t}}\left(-2 \mu \mathrm{x}_{0} \mathrm{x}_{0}(\mathrm{~s})(\mathrm{s}) \mathrm{x}_{3}(\mathrm{~s})+2 \mu \mathrm{x} \cdot 1 \mathrm{x}_{0}(\mathrm{~s})(\mathrm{s}) \mathrm{x}_{2}(\mathrm{~s})+\right.$

$\mu \mathrm{x}_{1}(\mathrm{~s})^{2} \mathrm{x}_{1}(\mathrm{~s})+2 \mu \mathrm{x}_{0}(\mathrm{~s}) \mathrm{x}_{1}(\mathrm{~s}) \mathrm{x}_{1}(\mathrm{~s}) \mathrm{x}_{2}(\mathrm{~s})-\mu \mathrm{x}_{3}(\mathrm{~s})+$

$\left.\mu \mathrm{x}_{0}^{2}(\mathrm{~s}) \mathrm{x}_{3}(\mathrm{~s})+\mathrm{x}_{3}(\mathrm{~s})\right) \mathrm{dsdt}=\frac{17}{58968} \mu^{4} \mathrm{t}^{13}+$

$\frac{1}{3991680}\left(13820 \mu^{4}+5265 \mu^{3}\right) \tau^{12}+$

$\frac{1}{3326400}\left(45892 \mu^{4}+49032 \mu 3+5625 \mu^{2}\right) \tau^{11}+$

$$
\begin{aligned}
& \frac{1}{3991680}\left(1320 \mu^{4}+5265 \mu^{3}\right) \tau^{12}+\frac{1}{3326400}\left(\begin{array}{c}
45892 \mu^{4}+ \\
49032 \mu^{3}
\end{array}\right)+ \\
& \frac{1}{217780}\left(-6720 \mu^{4}+1544 \mu^{3}+134136 \mu^{2}+10884 \mu+6\right) \tau^{9}+ \\
& \frac{1}{1693440}\left(-22848 \mu^{4}-7056 \mu^{3}+127512 \mu^{2}+25032 \mu+42\right) \tau^{8}+ \\
& \frac{1}{1270080}\left(-50400 \mu^{3}+12096 \mu^{2}+16128 \mu\right) \tau^{7}-\frac{1}{60} \mu^{2} t^{6}
\end{aligned}
$$

Consequently, the general solution of Eq. 34 is given as follow (Fig. 3):

$$
\begin{aligned}
& x(t)=x 0(t)+x 1(t)+x 2(t)+x 3(t)+\ldots,+x k(t)+, \ldots,= \\
& \left.1+\mathrm{t}-\frac{1}{12} \mu \mathrm{t}^{4}-\left(\frac{\mu}{3}+\frac{1}{6}\right) \mathrm{t}^{3}-\frac{1}{2} \mathrm{t}^{2}+\frac{1}{84} \mu^{2} \tau^{7}+\frac{1}{360}\left(30 \mu^{2}\right)+11 \mu\right) \tau^{6}+ \\
& \frac{1}{240}\left(32 \mu^{2}+4 \mu \mu+2\right) \tau^{5}+\frac{1}{144}(36 \mu+6) \tau^{4}+\frac{19}{10080} \mu^{3} \tau^{0}- \\
& \frac{1}{120960}\left(2280 \mu^{3}+804 \mu^{2}\right) \tau^{9} \frac{1}{94080}\left(5488 \mu^{3}+5628 u^{2}+47 \mu-\tau^{8}-\right. \\
& \frac{1}{70560}\left(3808 \mu^{3}+11760 \mu^{2}+2856 \mu+14\right) \tau^{7}-\frac{1}{50400}\left(7009 \mu^{2}+4620 \mu+70\right) \tau^{6}- \\
& \frac{1}{20} \mu \tau^{5}+\frac{17}{58968} \mu^{4} \tau^{3}+\frac{1}{3991680}\left(13820 \mu^{4}+5265 \mu^{3}\right) \tau^{2}+ \\
& \frac{1}{3326400}\left(45892 \mu^{4}+49032 \mu^{3}+5625 \mu^{2}\right) \tau^{11}+ \\
& \left(49884 \mu^{4}+150066 \mu^{3}+48177 \mu^{2}+1371 \mu\right) \tau^{10}+ \\
& \frac{1}{2177280}\left(-6720 \mu^{4}+154464 \mu^{3}+134136 \mu^{2}+10884 \mu+6\right) \tau^{9}+ \\
& \frac{1}{1693440}\left(-22818 \mu^{4}-7056 \mu^{3}+127512 \mu^{2}+25032 \mu+42\right) \tau^{8}+ \\
& \left.\frac{1}{1270080}\left(-50400 \mu^{3}+12096 \mu^{2}+16128 u\right)\right) \tau^{7}-\frac{1}{60} \mu^{2} \tau^{6}+\ldots
\end{aligned}
$$

Problem 4 (non linear Lienard equation): Consider the following non linear second-order ODE:

$$
u^{\prime \prime}(t)+u^{2}(t)=0
$$

Subject to initial conditions:

$$
\mathrm{u}(0)=\xi, \quad \mathrm{u}(0)=0
$$

Comparing Eq. 36, we have:

$$
\begin{aligned}
& f(u)=0, g(u)=u^{2}(t)=b_{2} u^{2}, \\
& b_{2}=1 \quad i=2 \text { and } h(t)=0
\end{aligned}
$$

The initial approximation has the form: 


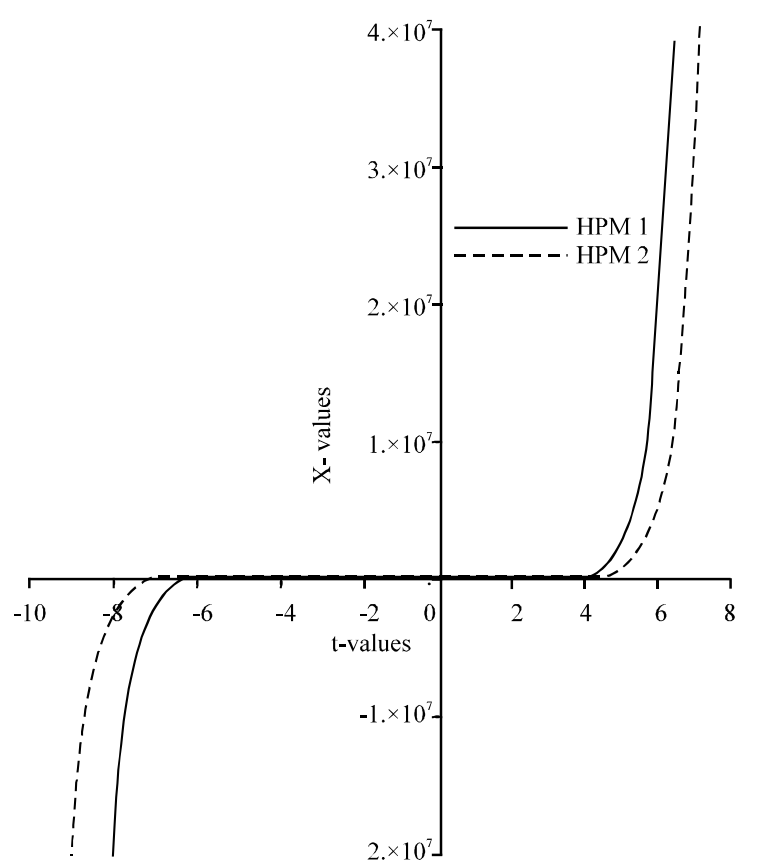

Fig. 3: Graphs of the approximated solution $x(t)$ versustimer for the values $\mathrm{m}=0.9$, lusing proposed HPM

$$
\mathrm{u}_{0}(\mathrm{t})=\xi
$$

Substituting Eq. 23 into Eq. 36, we have:

$\mathrm{u}_{1}(\mathrm{t})=-\iint_{\mathrm{t}}\left(\mathrm{u}_{0}(\mathrm{~s})+\mathrm{u}_{0}^{2}(\mathrm{~s})\right) \mathrm{ds} \mathrm{dt}=\frac{-1}{2 !} \xi^{2} \mathrm{t}^{2}$

$\mathrm{u}_{2}(\mathrm{t})=-\iint_{\mathrm{t}}\left(2 \mathrm{u}_{0}(\mathrm{~s}) \mathrm{u}_{1}(\mathrm{~s})\right) \mathrm{ds} d \mathrm{t}=\frac{2}{4 !} \xi^{3} \mathrm{t}^{4}$

$\mathrm{u}_{3}(\mathrm{t})=-\iint_{\mathrm{t}}\left(2 \mathrm{u}_{0}(\mathrm{~s}) \mathrm{u}_{2}(\mathrm{~s})+\mathrm{u}_{1}^{2}(\mathrm{~s}) \mathrm{ds} \mathrm{dt}=-\frac{10}{6 !} \xi^{4} \mathrm{t}^{6}\right.$

$\mathrm{u}_{4}(\mathrm{t})=-\iint_{\mathrm{t}}\left(2 \mathrm{u}_{0}(\mathrm{~s}) \mathrm{u}^{3}(\mathrm{~s})+2 \mathrm{u}_{1}(\mathrm{~s})\right) \mathrm{ds} d \mathrm{dt}=\frac{80}{8 !} \xi^{5} \mathrm{t}^{8}$

$u_{5}(t)=-\iint_{t}\left(2 u_{0}(s) u_{4}(s)+2 u_{1}(s) u_{3}(s)+u_{2}^{2}(s)\right) d s d t=-\frac{1000}{10 !} \xi^{6} t^{10}$

$\mathrm{u}_{6}(\mathrm{t})=-\iint_{\mathrm{t}}\left(2 \mathrm{u}_{0}(\mathrm{~s}) \mathrm{u}_{5}(\mathrm{~s})+2 \mathrm{u}_{1}(\mathrm{~s}) \mathrm{u}_{4}(\mathrm{~s})+2 \mathrm{u}_{2}(\mathrm{~s})\right) \mathrm{ds} d \mathrm{dt}=\frac{17600}{12 !} \xi^{7} \mathrm{t}^{12}$

Hence, we get the general solution of Eq. 36 is given as follow (Fig. 4):

$$
\begin{aligned}
& x(t)=x_{0}(t)+x_{1}(t)+x_{2}(t)+x_{3}(t)+, \ldots,+x_{k}(t)+, \ldots,= \\
& \xi-\frac{1}{2 !} \xi^{2} \tau^{2}+\frac{2}{4 !} \xi^{3} \tau^{4}-\frac{10}{6 !} \xi^{4} \tau^{6}+\frac{80}{8 !} \xi^{5} \tau^{8}- \\
& \frac{1000}{10 !} \xi^{6} \tau^{10}+\frac{17600}{12 !} \xi^{7} \tau^{12}+\ldots,
\end{aligned}
$$

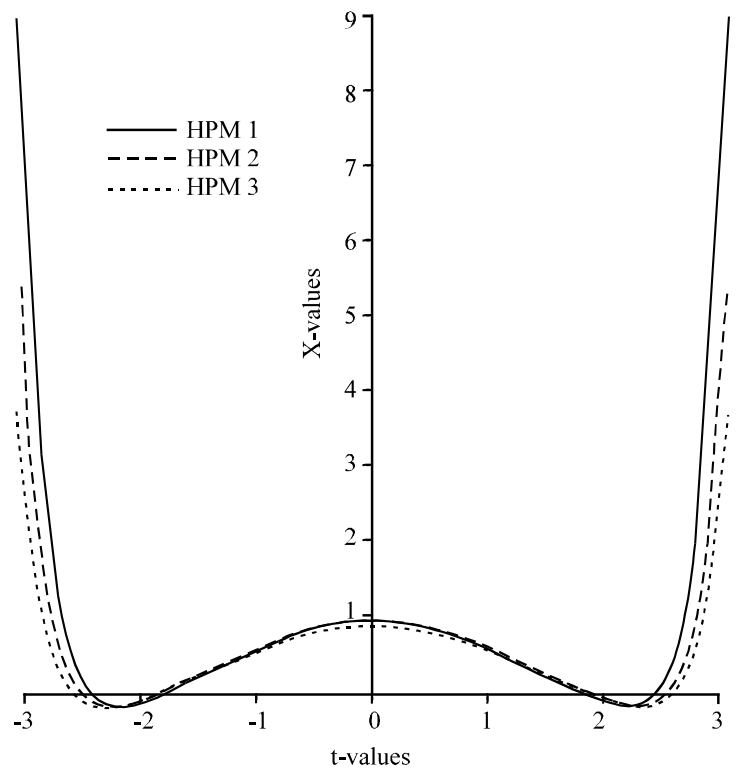

Fig. 4: Graphs of the approximated solution $x(t)$ versustimer for the values $=1 ;_{s}=0.94$ and $_{s}=0.9$ for solution using proposed HPM

Problem 5 (non linear lienard equation): Consider the non linear Lienard equation: (Harko et al., 2014):

$$
x^{\prime \prime}(t)+(a x(t)+b) x(t)+c x(t)+d-x^{3}(t)=0
$$

Subject to initial conditionis:

$$
x(0)=0, \quad x(0)=b
$$

Comparing Eq. 38, we have:

$$
\begin{array}{ll}
f(x)=a x+b, & g(x)=d+c x(t)-x_{3}, \quad i=0,1 \\
b_{0}=d \quad b_{1}=c & b 3=-1 \quad i=0,1,3 \text { and } h(t)=0
\end{array}
$$

The initial approximation has the form:

$$
x 0(t)=\beta t
$$

Substituting Eq. 23 into Eq. 39, we have:

$$
\begin{aligned}
& \left.\mathrm{x}_{1}(\mathrm{t})=-\iint_{\mathrm{t}} \mathrm{x}_{0}(\mathrm{~s})+\mathrm{bx_{0 }}(\mathrm{s})+\mathrm{ax}_{0}(\mathrm{~s}) \dot{\mathrm{x}}_{0}+\mathrm{d}+\mathrm{cx}_{0}(\mathrm{~s})-\mathrm{x}_{0}^{3}(\mathrm{~s})\right) \\
& \mathrm{ds} \mathrm{dt}=\frac{1}{20} \beta^{3} \tau^{5}-\beta\left(\frac{1}{6} \alpha \beta+\frac{\mathrm{c}}{6}\right) \tau^{3}+\left(\frac{-\mathrm{b}}{2} \beta-\frac{\mathrm{d}}{2}\right) \tau^{2} \\
& \mathrm{x}_{2}(\mathrm{t})=-\iint_{\mathrm{t}}\left(\dot{\mathrm{x}}_{1}(\mathrm{~s})+\mathrm{ax}_{0}(\mathrm{~s}) \dot{\mathrm{x}}_{1}+\mathrm{ax}_{1}(\mathrm{~s}) \dot{\mathrm{x}}_{0}+\right. \\
& \left.\mathrm{cx}_{1}(\mathrm{~s})-3 \mathrm{x}_{0}^{2}(\mathrm{~s}) \mathrm{x}_{1}(\mathrm{~s})\right) \mathrm{ds} \mathrm{dt}=\frac{1}{480} \beta^{5} \mathrm{t}^{9}+
\end{aligned}
$$




$$
\begin{aligned}
& \left(-\frac{1273}{151200} a^{2} b \beta^{5}-\frac{79}{12096} a^{2} \beta^{4} d-\frac{1397}{201600} a b \beta^{4} c-\frac{2987}{604800} a \beta^{3} c d-t_{t^{10}+}\right. \\
& \frac{7}{3600} \mathrm{~b} \beta^{5}-\frac{41}{43200} \mathrm{~b} \beta^{3} \mathrm{c}^{2}-\frac{1}{600} \beta^{4} \mathrm{~d}-\frac{389}{604800} \beta^{2} \mathrm{c}^{2} \mathrm{~d} \\
& \left(\frac{31}{22680} a^{4} b^{5}+\frac{97}{36288} a^{3} \beta^{4} c+\frac{1}{720} a^{2} \beta^{5}+\frac{31}{20160} a^{2} \beta^{3} c^{2}-\frac{5}{288} a b^{2} \beta^{4}-\right) \\
& \frac{1439}{60480} a b \beta^{3} d+\frac{1}{576} \alpha \beta^{4} c+\frac{17}{72576} a \beta^{2} c^{3}-\frac{11}{2160} b^{2} \beta^{3} c-\frac{19}{2520} a \beta^{2} d^{2}-t^{9}+ \\
& \frac{379}{60480} \mathrm{~b} \beta^{2} \mathrm{~cd}+\frac{1}{2880} \beta^{3} \mathrm{c}^{2}+\frac{1}{362880} \beta \mathrm{c}^{4}-\frac{1}{504} \beta \mathrm{od}^{2} \\
& \left(\frac{31}{2520} \mathrm{a}^{3} \mathrm{~b} \beta^{4}+\frac{13}{1440} \alpha^{3} \beta^{3} d+\frac{101}{6720} \mathrm{a}^{2} b \beta^{3} \mathrm{c}+\frac{11}{1120} \mathrm{a}^{2} \beta^{2} \alpha d+\frac{1}{112} \mathrm{ab} \beta^{4}+\right. \\
& \frac{9}{2240} a b \beta^{2} c^{2}-\frac{31}{3360} b^{3} \beta^{3}+\frac{3}{448} a \beta^{3} d+\frac{9}{4480} a \beta c^{2} d-\frac{53}{3360} d^{2} b^{2} d+\quad t^{3}+ \\
& \frac{1}{224} \mathrm{~b} \beta^{3} \mathrm{c}+\frac{1}{10080} \mathrm{~b} \beta \mathrm{c}^{3}-\frac{1}{112} \mathrm{~b} \beta \mathrm{d}^{2}+\frac{1}{448} \beta^{2} \mathrm{~cd}+\frac{1}{40320} \mathrm{c}^{3} d-\frac{1}{448} \mathrm{~d}^{3} \\
& \left(\frac{1}{28} a^{2} b^{2} \beta^{3}+\frac{19}{420} a^{2} b \beta^{2} d+\frac{3}{140} a b^{2} \beta^{2} c+\frac{1}{80} a^{2} \beta d^{2}+\frac{3}{140} a b \beta c d+\right) \\
& \frac{1}{84} b^{2} \beta^{3}+\frac{1}{840} b^{2} \beta c^{2}+\frac{1}{280} a^{2}+\frac{3}{140} a b \beta o d+\frac{1}{84} b^{2} \beta^{3}+\quad t^{6}+ \\
& \frac{1}{840} b^{2} \beta c^{2}+\frac{1}{180} b^{3} \beta c+\frac{13}{720} a b d^{2}+\frac{1}{240} b^{2} c d \\
& \left(\frac{1}{120} b^{4} \beta+\frac{1}{120} b^{3} d\right) t^{5}
\end{aligned}
$$

Then, the general solution of Eq. 38 is written as follow (Fig. 5):

$$
\begin{aligned}
& \mathrm{x}(\mathrm{t})=\mathrm{x}_{0}(\mathrm{t})+\mathrm{x}_{1}(\mathrm{t})+\mathrm{x}_{2}(\mathrm{t})+\mathrm{x}_{3}(\mathrm{t})+\mathrm{x}_{4}(\mathrm{t})+, \ldots,=\beta \mathrm{t}+1 \beta^{3} \mathrm{t}^{5}- \\
& \beta(1 \alpha \beta+\mathrm{c}) \mathrm{t}^{3}+(-1 \mathrm{~b} \beta-1 \mathrm{~d}) \mathrm{t}^{2}+\left(-\frac{2}{105} \mathrm{a} \beta 4-\frac{11}{840} \beta^{3} \mathrm{c}\right) \mathrm{t}^{7}+ \\
& \left(-\frac{7}{120} \mathrm{~b} \beta^{3}-\frac{1}{20} \beta^{2} \mathrm{~d}\right) \mathrm{t}^{6}+\left(\frac{1}{30} \mathrm{a}^{2} \beta^{3}+\frac{1}{24} \mathrm{a} \beta^{2} \mathrm{c}+\frac{1}{120} \beta \mathrm{c}^{2}\right) \mathrm{t}^{5}+ \\
& \left(\frac{1}{6} \mathrm{ab} \beta^{2}+\frac{1}{8} \mathrm{a} \beta d+\frac{1}{12} \mathrm{~b} \beta \mathrm{c}+\frac{1}{24} \mathrm{~cd}\right) \mathrm{t}^{4}+\left(\frac{1}{6} \mathrm{~b}^{2} \beta+\frac{1}{6} \mathrm{bd}\right) \mathrm{t}^{3}+ \\
& \frac{11}{124800} \beta^{7} \mathrm{t}^{13}+\left(-\frac{59}{46200} \mathrm{a} \beta^{6}-\frac{307}{369600} \beta^{5} \mathrm{c}\right) \mathrm{t}^{11}+ \\
& \left(-\frac{11}{2880} \mathrm{~b} \beta 5-\frac{1}{300} \beta^{4} \mathrm{~d}\right) \mathrm{t}^{10}+\left(\frac{17}{30240} \mathrm{a}^{2} \beta^{5}+\frac{3}{448} \mathrm{a} \beta^{4} \mathrm{c}+\right. \\
& \left(\frac{103}{3360} \mathrm{ab} \beta^{4}+\frac{1}{40} \mathrm{a} \beta^{3} \mathrm{~d}+\frac{9}{560} \mathrm{~b} \beta^{3} \mathrm{c}^{3}+\frac{27}{2240} \beta^{2} \mathrm{~cd}\right) \mathrm{t}^{9}+ \\
& \left(-\frac{17}{2520} \mathrm{t}^{3} \beta^{4}-\frac{3}{280} \mathrm{a}^{2} \beta^{3} \mathrm{c} \frac{1}{240} \mathrm{a} \beta^{2} \mathrm{c}^{2}+\frac{4}{105} \mathrm{~b}^{2} \beta^{3}+\right. \\
& \frac{23}{420} \mathrm{~b} \beta^{2} \mathrm{~d} \frac{1}{5040} \mathrm{bc} \mathrm{c}^{3}+\frac{1}{56} \beta \mathrm{d}^{2}
\end{aligned}
$$




$$
\begin{aligned}
& \left(-\frac{17}{360} a^{2} b \beta^{3}-\frac{5}{144} a^{2} \beta^{2} d-\frac{29}{720} a b \beta^{2} c-\frac{1}{40} a \beta o d-\frac{1}{240} b \beta c^{2}-\frac{1}{720} c^{2} d\right) t^{6}+ \\
& \left.\left(-\frac{11}{120} a b^{2} \beta^{2}-\frac{13}{120} a b \beta d-\frac{1}{40} b^{2} \beta c-\frac{1}{40} a d^{2}-\frac{b o d}{60}\right)\right) t^{5}+\left(-\frac{1}{24} b^{3} \beta-\frac{1}{24} b^{2} d\right) t^{4}+ \\
& \frac{81}{56576000} \beta^{9} \mathrm{t}^{17}+\left(\begin{array}{c}
-\frac{83}{2242240} \mathrm{a} \beta^{8}- \\
\frac{7361}{403603200} \beta^{7} \mathrm{c}
\end{array}\right) \mathrm{t}^{15}+\left(-\frac{157}{1747200} \mathrm{~b} \beta^{7}-\frac{11}{145600} \beta^{6} \mathrm{~d}\right) \mathrm{t}^{14} \\
& +\left(\frac{28747}{86486400} \mathrm{a}^{2} \beta^{7}+\frac{3821}{11531520} \mathrm{a} \beta^{6} \mathrm{c}+\frac{1}{24960} \beta^{7}+\frac{59}{915200} \beta^{5} \mathrm{c}^{2}\right) \mathrm{t}^{13}+ \\
& \left(\frac{2183}{1330560} \mathrm{ab} \beta^{6}+\frac{281}{211200} \mathrm{a} \beta^{5} \mathrm{~d} \frac{4343}{6652800} \mathrm{~b} \beta^{5} \mathrm{c}+\frac{197}{4032} \beta^{4} \mathrm{~cd}\right) \mathrm{t}^{12}+ \\
& \left(\begin{array}{l}
-\frac{31}{26400} a^{3} \beta^{6}-\frac{1459}{831600} a^{2} \beta^{5} c-\frac{1}{1925} a \beta^{6}-\frac{1531}{2217600} a \beta^{4} c^{2}+ \\
\frac{383}{221760} b^{2} b^{5}+\frac{229}{92400} b \beta^{4} d-\frac{1}{2800} \beta^{5} c-\frac{19}{302400} \beta^{3} c^{3}+\frac{51}{61600} \beta^{3} d^{2}
\end{array}\right) t^{11}+ \\
& \left(-\frac{1273}{151200} a^{2} b \beta^{5} \frac{79}{12096} a^{2} \beta^{4} d-\frac{1379}{201600} a b \beta^{4} c-\frac{2987}{604800} a \beta^{3} c d-\right)_{t^{10}+} \\
& \frac{7}{3600} \mathrm{~b} \beta^{5}-\frac{41}{43200} \mathrm{~b} \beta 3 \mathrm{c}^{2}-\frac{1}{600} \beta^{4} \mathrm{~d}-\frac{389}{604800} \beta^{2} \mathrm{c}^{2} \mathrm{~d} \\
& \left(\frac{31}{22680} a \beta^{5}+\frac{97}{36288} a^{3} \beta^{4} c+\frac{1}{720} a^{2} \beta^{5}+\frac{31}{20160} a^{2} \beta^{3} c^{2}-\frac{5}{288} a b^{2} \beta^{4}-\right) \\
& \frac{1439}{60480} a b \beta^{3} d+\frac{1}{576} a \beta^{4} c+\frac{17}{72576} a \beta^{2} c^{3}-\frac{11}{2160} b^{2} \beta^{3} c-\frac{19}{2520} a \beta^{2 d 2}-t^{9}+ \\
& \frac{379}{60480} \mathrm{~b} \beta^{2} \mathrm{~cd}+\frac{1}{1880} \beta^{3} \mathrm{c}^{2}+\frac{1}{36288} \beta \mathrm{c}^{4}-\frac{1}{504} \beta \mathrm{od}^{2} \\
& \left(\frac{31}{2520} a^{3} b \beta^{4}+\frac{13}{1440} a^{3} \beta^{3} d+\frac{101}{6720} a^{2} b \beta^{3} c+\frac{11}{1120} a^{2} \beta^{2} \alpha d+\frac{1}{112} a b \beta^{4}+\right. \\
& \frac{9}{2240} a b \beta^{2} c^{2}-\frac{31}{3360} b^{3} \beta^{3}+\frac{3}{448} a \beta^{3} d+\frac{9}{4480} a b c^{2} d-\frac{53}{3360} b^{2} \beta^{2} d+\quad t^{8}+ \\
& \frac{1}{224} \mathrm{~b} \beta^{3} \mathrm{c}+\frac{1}{10080} \mathrm{~b} \beta \mathrm{c}^{3}-\frac{1}{112} \mathrm{~b} \beta \mathrm{d}^{2}+\frac{1}{448} \beta^{2} \alpha \mathrm{d}+\frac{1}{40320} \mathrm{c}^{3} \mathrm{~d}-\frac{1}{448} \mathrm{~d}^{3} \\
& \left(\begin{array}{l}
\frac{1}{28} a^{2} b^{2} \beta^{3}+\frac{19}{420} a^{2} b \beta^{2} d+\frac{3}{140} a b^{2} \beta^{2} c+\frac{1}{80} a^{2} \beta d^{2}+\frac{3}{140} a b \beta c d+ \\
\frac{1}{84} b^{2} \beta^{3}+\frac{1}{840} b^{2} \beta c^{2}+\frac{1}{280} a d d^{2}+\frac{1}{84} b \beta^{2} d+\frac{1}{1680} b c^{2} d
\end{array}\right) t^{7}+ \\
& \left(\frac{13}{360} a b^{3} \beta^{2}+\frac{19}{360} a b^{2} \beta d+\frac{1}{180} b^{3} \beta c+\frac{13}{720} a b d^{2}+\frac{1}{240} b^{2} c d\right) t^{6}+ \\
& \left(\frac{1}{120} b^{4} \beta+\frac{1}{120} b^{3} d\right) t^{5}+\ldots
\end{aligned}
$$

Problem 6 (non linear lienardequation): We will study the following non-linear Lienard equation (Matinfar et al., 2008; Harko et al., 2014):

$$
\mathrm{x}^{\prime \prime}(\mathrm{t})+\mathrm{Lx}(\mathrm{t})+\mathrm{Mx}^{3}(\mathrm{t})+\mathrm{Nx^{5 }}(\mathrm{t})=0
$$

Subject to initial conditions:

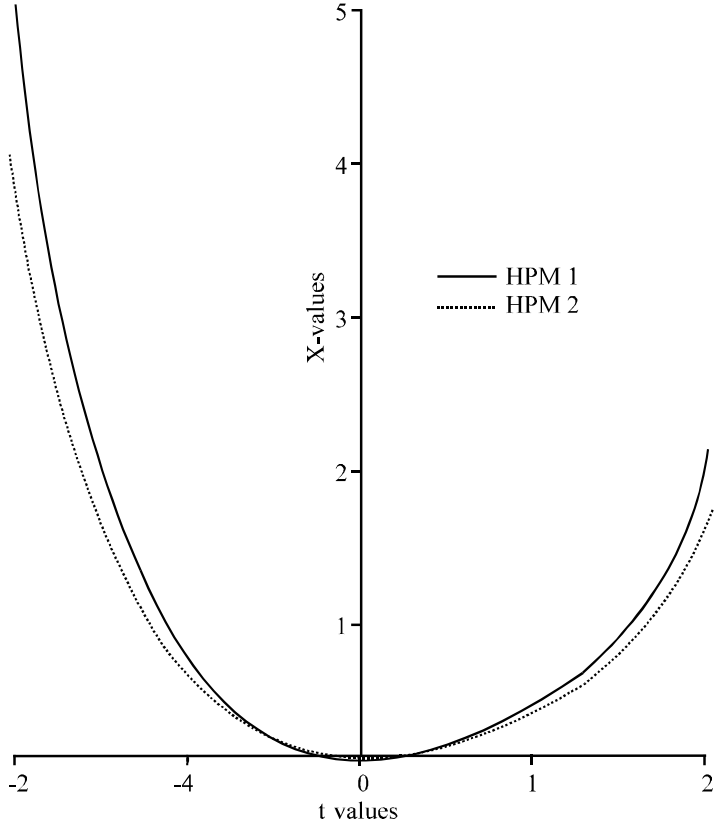

Fig. 5: Graphs of the approximated solution $\mathrm{x}(\mathrm{t})$ versus time $\mathrm{t}$ for the values $\mathrm{a}=\mathrm{b}=\mathrm{c}=1 ; \beta=0$ and $\mathrm{a}=$ $0.9 ; \mathrm{b}=\mathrm{c}=0.8 ; \mathrm{s}=0 ; \mathrm{d}=-0.9$ using proposed HPM

$$
\mathrm{x}(0)=\alpha, \mathrm{x}(0)=0
$$

Comparing Eq. 40, we have:

$$
\begin{aligned}
& \mathrm{f}(\mathrm{x})=0 ; \mathrm{g}(\mathrm{x})=\mathrm{Lx}(\mathrm{t})+\mathrm{Nx^{5 }}(\mathrm{t}) \text { and } \mathrm{h}(\mathrm{t})=0 \\
& \mathrm{~b}_{1}=\mathrm{L} ; \mathrm{b}_{3}=\mathrm{M} ; \mathrm{b}_{5}=\mathrm{N} ; \mathrm{i}=1,3,5
\end{aligned}
$$

The initial approximation has the form:

$$
x_{0}(t)=\alpha
$$

Substituting Eq. 23 into Eq. 40, we have:

$$
\begin{aligned}
& \mathrm{x}_{1}(\mathrm{t})=-\iint_{\mathrm{t}}\left(\mathrm{x}_{0}(\mathrm{~s})+\mathrm{Lx} \mathrm{x}_{0}(\mathrm{~s})+\mathrm{Mx}_{0}^{3}+\mathrm{Nx}_{0}^{5}(\mathrm{~s})\right) \mathrm{ds} \mathrm{dt}= \\
& \left(-\frac{1}{2} \mathrm{~N} \alpha^{5} \mathrm{M} \alpha^{3}-\frac{1}{2} \mathrm{~L} \alpha\right) \mathrm{t}^{2} \\
& \mathrm{x}_{2}(\mathrm{t})=-\iint_{\mathrm{t}}\left(\mathrm{Lx}_{1}(\mathrm{~s})+3 \mathrm{Mx}_{0}^{2}(\mathrm{~s}) \mathrm{x}_{1}(\mathrm{~s})+5 \mathrm{Nx}_{0}^{4}(\mathrm{~s}) \mathrm{x}_{1}(\mathrm{~s})\right) \mathrm{ds} \mathrm{dt}= \\
& \frac{1}{24} \mathrm{t}^{2} \alpha\left(5 \mathrm{~N} \alpha^{4}+3 \mathrm{M} \alpha^{2}+\mathrm{L}\right)\left(\mathrm{N} \alpha^{4}+\mathrm{M} \alpha^{2}+\mathrm{L}\right) \\
& \mathrm{x}_{3}(\mathrm{t})=-\iint_{\mathrm{t}}\left(\mathrm{Lx}_{2}(\mathrm{~s})+3 \mathrm{Mx}_{0}(\mathrm{~s}) \mathrm{x}_{1}^{2}(\mathrm{~s})+3 \mathrm{Mx}_{0}^{2}(\mathrm{~s}) \mathrm{x}_{2}(\mathrm{~s})\right. \\
& \left.+5 \mathrm{Nx}_{0}^{4}(\mathrm{~s}) \mathrm{x}_{2}(\mathrm{~s})+10 \mathrm{Nx}_{0}^{3}(\mathrm{~s}) \mathrm{x}_{0}^{3}(\mathrm{~s})\right) \mathrm{ds} \mathrm{dt}=-\frac{1}{720} \mathrm{t}^{6} \alpha+ \\
& \left.\mathrm{M} \alpha^{2}+\mathrm{L}\right)\left(85 \mathrm{~N}^{2} \alpha^{8}+108 \mathrm{MN} \alpha^{6}+70 \mathrm{LN} \alpha^{4}+27 \mathrm{M}^{2} \alpha^{4}+24 \mathrm{LM} \alpha^{2}+\mathrm{L}^{2}\right) \\
& \mathrm{x}_{4}(\mathrm{t})=-\iint_{\mathrm{t}}\left(\mathrm{Lx}_{3}(\mathrm{~s})+3 \mathrm{Mx}_{0}^{2}(\mathrm{~s}) \mathrm{x}_{3}(\mathrm{~s})+6 \mathrm{Mx}_{0}(\mathrm{~s}) \mathrm{x}_{1}(\mathrm{~s}) \mathrm{x}_{2}(\mathrm{~s})+\right.
\end{aligned}
$$


$\mathrm{Mx}_{1}(\mathrm{~s})^{3}+5 \mathrm{Nx}_{0}^{4}(\mathrm{~s}) \mathrm{x}_{3}(\mathrm{~s})+20 \mathrm{Nx}_{0}^{3}(\mathrm{~s}) \mathrm{x}_{1}(\mathrm{~s}) \mathrm{x}_{2}(\mathrm{~s})+$

$\left.10 \mathrm{Nx}_{0}^{2}(\mathrm{~s}) \mathrm{x}_{1}^{2}(\mathrm{~s})\right) \mathrm{ds} \mathrm{dt}=\frac{1}{720} \alpha\left(\mathrm{N} \alpha^{4}+\mathrm{M}^{2}+\mathrm{L}\right)$

$\left(\begin{array}{l}\frac{275}{8} \mathrm{~N}^{3} \alpha^{12}+\frac{3735}{56} \mathrm{MN}^{2} \alpha^{10}+\frac{2235}{56} \mathrm{LN}^{2} \alpha^{8}+ \\ \frac{2259}{56} \mathrm{M}^{2} \mathrm{~N} \alpha^{8}+\frac{1179}{28} \mathrm{LMN} \alpha^{6}+\frac{63}{8} \mathrm{M}^{3} \alpha^{6}+ \\ \frac{375}{56} \mathrm{~L}^{2} \mathrm{~N} \alpha^{4}+\frac{639}{56} \alpha^{4} \mathrm{M}^{2} \mathrm{~L}+\frac{207}{56} \mathrm{~L}^{2} \mathrm{M} \alpha^{2}+\frac{1}{56} \mathrm{~L}^{3}\end{array}\right) \mathrm{t}^{8}+$

$\frac{1}{720} \alpha\left(N \alpha^{4}+M \alpha^{2}+L\right)\left(-60 N^{2} \alpha^{7}-60 M N \alpha^{5}-60 L N \alpha^{3}\right) t^{6}$

and

$\mathrm{X}_{4}(\mathrm{t})=-\iint_{\mathrm{t}}\left(\left(\mathrm{Lx}_{4}(\mathrm{~s})+3 \mathrm{Mx}^{2}(\mathrm{~s}) \mathrm{x}_{4}(\mathrm{~s})+6 \mathrm{Mx}_{0}(\mathrm{~s}) \mathrm{x}_{1}(\mathrm{~s}) \mathrm{x}_{3}(\mathrm{~s})\right.\right.$

$+3 \mathrm{Mx}_{1}(\mathrm{~s})^{2} \mathrm{x}_{2}+3 \mathrm{Mx}_{0}(\mathrm{~s}) \mathrm{x}^{2}+5 \mathrm{Nx}_{0}^{4}(\mathrm{~s}) \mathrm{x}_{4}(\mathrm{~s})+$

$20 \mathrm{Nx}_{0}^{3}(\mathrm{~s}) \mathrm{x}_{1}(\mathrm{~s}) \mathrm{x}_{3}(\mathrm{~s})+30 \mathrm{Nx}_{0}^{2}(\mathrm{~s}) \mathrm{x}_{1}^{2}(\mathrm{~s}) \mathrm{x}_{2}+10 \mathrm{Nx}_{0}^{3}(\mathrm{~s}) \mathrm{x}_{0}^{2}(\mathrm{~s})+$

$\left.5 \mathrm{Nx}_{0}(\mathrm{~s}) \mathrm{x}_{1}^{4}(\mathrm{~s})\right) \mathrm{ds} \mathrm{dt}=-\frac{1}{140320} \alpha\left(\mathrm{nN} \alpha^{4}+\mathrm{M} \alpha^{2}+\mathrm{L}\right) \frac{30065}{18} \mathrm{~N}^{4} \alpha^{16}+$

$\frac{13282}{3} \mathrm{MN}^{3} \alpha^{14}+\frac{30080}{9} \mathrm{LN}^{3} \alpha^{12}+\frac{60359}{15} \mathrm{M}^{2} \mathrm{~N}^{2} \alpha^{12}+\frac{17240}{3} \mathrm{LMN}^{2} \alpha^{10}+$

$\frac{6974}{5} \mathrm{M}^{3} \mathrm{~N} \alpha^{10}+1973 \mathrm{~L}^{2} \mathrm{~N}^{2} \alpha^{8}+\frac{13548}{5} \mathrm{LM}^{2} \mathrm{~N} \alpha^{8}+\frac{1281}{10} \mathrm{M}^{4} \alpha^{8}+$

$\frac{8182}{5} \mathrm{~L}^{2} \mathrm{MN} \alpha^{6}+\frac{1272}{5} \mathrm{LM}^{3} \alpha^{6}+\frac{2684}{9} \mathrm{~L}^{3} \mathrm{~N} \alpha^{4}+147 \Lambda^{2} \mathrm{M}^{2} \alpha^{4}+$

$\left.\frac{308}{15} \mathrm{~L}^{3} \mathrm{M} \alpha^{2}+\frac{1}{90} \mathrm{~L}^{4}\right) \mathrm{t}^{10}-\frac{1}{40320} \alpha\left(\mathrm{N \alpha}^{4}+\mathrm{M \alpha}^{2}+\mathrm{L}\right)$

$\left(\begin{array}{l}-300 \mathrm{~N}^{3} \alpha^{11}-480 \mathrm{MN}{ }^{2} \alpha^{9}-360 \mathrm{LN}^{2} \alpha^{7} \\ -180 \mathrm{M}^{2} \mathrm{~N} \alpha^{7}-240 \mathrm{LMN} \alpha^{5}-60 \mathrm{~L}^{2} \mathrm{~N} \alpha^{3}\end{array}\right) \mathrm{t}^{8}$

Therefore, we get the general solution (Fig. 6):

$$
\begin{aligned}
& x(t)=x_{0}(t)+x_{1}(t)+x_{2}(t)+x_{3}(t)+x_{4}(t)+x_{5}(t)+, \ldots
\end{aligned}
$$

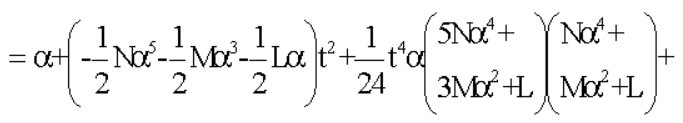

$$
\begin{aligned}
& -\frac{1}{720} \mathrm{t}^{6} \alpha\left(\mathrm{No} \alpha^{4}+\mathrm{M} \alpha^{2}+\mathrm{L}\right)\left(\begin{array}{l}
85 \mathrm{~N}^{2} \alpha^{8}+108 \mathrm{MN} \alpha^{6}+70 \mathrm{LN \alpha} \alpha^{4}+ \\
27 \mathrm{M}^{2} \alpha^{4}+24 \mathrm{LM} \alpha^{2}+\mathrm{L}^{2}
\end{array}\right)+ \\
& +\frac{1}{720} \alpha\left(N \alpha^{4}+M \alpha^{2}+L\right)\left(\begin{array}{l}
\frac{275}{8} N^{3} \alpha^{12}+\frac{3735}{56} M N^{2} \alpha^{10}+\frac{2235}{56} L^{2} \alpha^{8}+ \\
\frac{2259}{56} M^{2} N \alpha^{8}+\frac{1179}{28} L M N \alpha^{6}+\frac{63}{8} M^{3} \alpha^{6}+ \\
\frac{375}{56} L^{2} N \alpha^{4}+\frac{639}{56} \alpha^{4} M^{2} L+\frac{207}{56} L^{2} M \alpha^{2}+\frac{1}{56} L^{3}
\end{array}\right) t^{8}+
\end{aligned}
$$

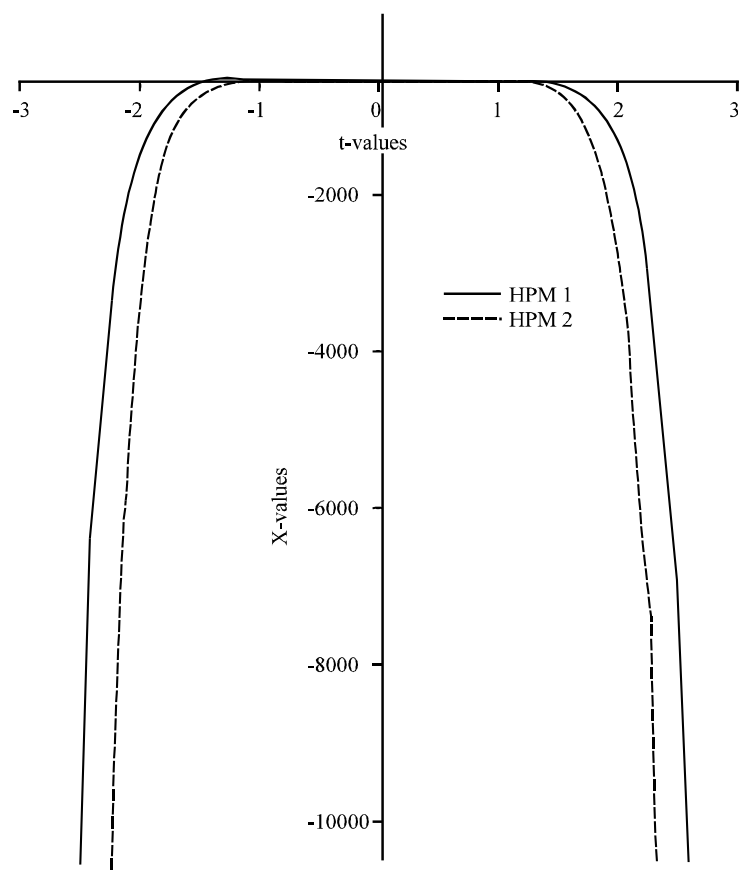

Fig. 6: Graphs of the approximated solution $\mathrm{x}(\mathrm{t})$ versus time $\mathrm{t}$ for the values of $s=\mathrm{N}=0.9 ; \mathrm{L}=\mathrm{M}=0.8$ and s $=1 ; \mathrm{L}=\mathrm{M}=\mathrm{N}=0.8$ using proposed HPM

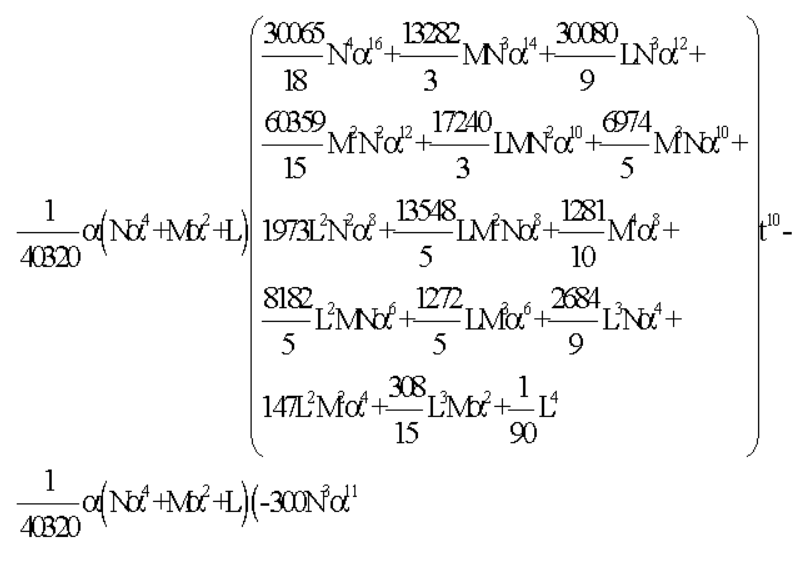

\section{CONCLUSION}

In this study, the Homotopy Perturbation Method (HPM) has been modified for solving gen- eralized linear \& nonlinear Liénard equation and the Duffing equation. The approximated solutions of this class of nonlinear equations have been derived using Maple. Also, we have tested the modified HPM on the solving of different implementations using MATLAB which are show the efficiency and accuracy of the proposed method. The approximated solutions of the problems 1-6 are agree well 
with analytical solutions for the tested problems Moreover, the approximated solutions proved that the proposed method to be efficient and high accurate.

\section{ACKNOWLEDGEMENT}

The researchers would like to thank University of Kufa for supporting this research project.

\section{REFERENCES}

Abbasbandy, S., 2006. Homotopy perturbation method for quadratic Riccati differential equation and comparison with Adomian's decomposition method. Appl. Math. Comput., 172: 485-490.

Abbasbandy, S., J.L. Lopez and R. Lopez-Ruiz, 2011. The homotopy analysis method and the Lienard equation. Intl. J. Comput. Math., 88: 121-134.

Al-Saif, A.J. and D.A. Abood, 2011. The homotopy perturbation method for solving $\mathrm{K}(2,2)$ equation. J. Basrah. Res. Sci., 37: 1-8.

Aswhad, A.A. and A.F. Jaddoa, 2016. The approximate solution of newell whitehead segel and fisher equations using the adomian decomposition method. Al Mustansiriyah J. Sci., 25: 1-12.

Babolian, E., A. Azizi and J. Saeidian, 2009. Some notes on using the homotopy perturbation method for solving time-dependent differential equations. Math. Comput. Modell., 50: 213-224.

Bataineh, A.S., M.S.M. Noorani and I. Hashim, 2009. Modi?ed homotopy analysis method for solving systems of second-order BVPs. Commun. Nonlinear Sci. Numer. Simul., 14: 430-442.

Batiha, B., 2015. A new efficient method for solving quadratic Riccati differential equation. Intl. J. Appl. Math. Res., 4: 24-24.

Chang, H.K. and J.C. Liou, 2006. Solving wave dispersion equation for dissipative media using homotopy perturbation technique. J. Waterway Port Coastal Ocean Eng., 132: 28-35.

Chun, C. and R. Sakthivel, 2010. Homotopy perturbation technique for solving two-point boundary value problems-comparison with other methods. Comput. Phys. Commun., 181: 1021-1024.

Fereidoon, A., H. Yaghoobi and M. Davoudabadi, 2011. Application of the homotopy perturbation method for solving the foam drainage equation. Intl. J. Differ. Equ., 2011: 1-13.

Gulkac, V., 2010. The homotopy perturbation method for the Black-Scholes equation. J. Stat. Comput. Simul., 80: 1349-1354.
Harko, T., F.S. Lobo and M.K. Mak, 2014. A class of exact solutions of the Lienard-type ordinary nonlinear differential equation. J. Eng. Math., 89: 193-205.

He, J.H., 1999. Homotopy perturbation technique. Comput. M ethods Applied Mech. Eng., 178: 257-262.

He, J.H., 2000. A coupling method of a homotopy technique and a perturbation technique for non-linear problems. Int. J. Non-Linear Mech., 35: $37-43$.

He, J.H., 2004. The homotopy perturbation method for nonlinear oscillators with discontinuities. Applied Math. Comput., 151: 287-292.

Jafari, H., H. Tajadodi and S.H. Matikolai, 2010. Homotopy perturbation pade technique for solving fractional Riccati differential equations. Intl. J. Non. Sci. Numer. Simul., 11: 271-276.

Jalaal, M., D.D. Ganji and F. Mohammadi, 2010. He's homotopy perturbation method for two-dimensional heat conduction equation: Comparison with finite element method. Heat Transfer Asian Res., 39: 232-245.

Javidi, M., 2009. Modified homotopy perturbation method for solving system of linear Fredholm integral equations. Math. Comput. Modell., 50: 159-165.

Kumar, S., O.P., Singh and S. Dixit, 2011. Generalized Abel inversion using Homotopy perturbation method. Appl. Math., 2: 254-257.

Liao, S., 2004. On the homotopy analysis method for nonlinear problems. Applied Mathe. Comput., 147: 499-513.

Ma, X., L. Wei and Z. Guo, 2008. He's homotopy perturbation method to periodic solutions of nonlinear Jerk equations. J. Sound Vib., 314: 217-227.

Matinfar, M., H. Hosseinzadeh and M. Ghanbari, 2008. A numerical implementation of the variational iteration method for the Lienard equation. World J. Modell. Simul., 4: 205-210.

Mechee, M., F. Ismail, Z.M. Hussain and Z. Siri, 2014. Direct numerical methods for solving a class of thirdorder partial differential equations. Appl. Math. Comput., 247: 663-674.

Mechee, M.S., A.M. Al-Rammahi and G.A. Al-Juaifri, 2017a. A study of general second-order partial differential equations using homotopy perturbation method. Global J. Pure Appl. Math., 13: 2471-2492.

Mechee, M.S., G.A. Al-Juaifri and A.K. Joohy, $2017 \mathrm{~b}$. Modified homotopy perturbation method for solving generalized linear complex differential equations. Appl. Math. Sci., 11: 2527-2540.

Momani, S. and Z. Odibat, 2007. Homotopy perturbation method for nonlinear partial differential equations of fractional order. Phys. Lett. A, 365: 345-350. 
Neamaty, A. and R. Darzi, 2010. Comparison between the variational iteration method and the homotopy perturbation method for the Sturm-Liouville differential equation. Boundary Value Prob., 2010: $1-14$.

Noor, M.A. and S.T. Mohyud-Din, 2008. Homotopy perturbation method for solving sixth-order boundary value problems. Comput. Math. Appl., 55: 2953-2972.

Odibat, Z. and S. Momani, 2008. Modified homotopy perturbation method: Application to quadratic Riccati differential equation of fractional order. Chaos Solitons Fractals, 36: 167-174.

Ozis, T. and C. Akci, 2011. Periodic solutions for certain non-smooth oscillators by iterated homotopy perturbation method combined with modified Lindstedt-Poincare technique. Meccanica, 46: 341-347.

Rafei, M., D.D. Ganji and H. Daniali, 2007. Solution of the epidemic model by homotopy perturbation method. Applied Math. Comput., 187: 10561062 .

Saberi-Nadjafi, J. and M. Tamamgar, 2008. Modified homotopy perturbation method for solving the system of Volterra integral equations. Intl. J. Nonlinear Sci. Numer. Simul., 9: 409-414.

Taghipour, R., 2010. Application of homotopy perturbation method on some linear and nonlinear periodic equations. World Appl. Sci. J., 10: $1232-1235$.
Vahidi, A.R., E. Babolian and Z. Azimzadeh, 2011. An improvement to the homotopy perturbation method for solving nonlinear Duffing's equations. Bull. Malaysian Math. Sci. Soc., 1: 1-13.

Wang, X. and X. Song, 2007. Global stability and periodic solution of a model for HIV infection of CD4+ T cells. Appl. Math. Comput., 189: 1331-1340.

Yazdi, A.A., 2013. Homotopy perturbation method for nonlinear vibration analysis of functionally graded plate. J. Vib. Acoust., 135: 021012-021018.

Yildirim, A. and D. Agirseven, 2009. The homotopy perturbation method for solving singular initial value problems. Intl. J. Nonlinear Sci. Numer. Simul., 10: 235-238.

Yildirim, A., 2009. On the solution of the nonlinear Korteweg-de Vries equation by the homotopy perturbation method. Intl. J. Numer. Methods Biomed. Eng., 25: 1127-1136.

Yildirim, A., 2010. He's homotopy perturbation method for nonlinear differential-difference equations. Intl. J. Comput. Math., 87: 992-996.

Yusufoglu, E., 2009. Improved homotopy perturbation method for solving Fredholm type integro-differential equations. Chaos Solitons Fractals, 41 : 28-37.

Zhou, S. and H. Wu, 2012. Analytical solutions of nonlinear Poisson-Boltzmann equation for colloidal particles immersed in a general electrolyte solution by homotopy perturbation technique. Colloid Polym. Sci., 290: 1165-1180. 